\title{
Global small data solutions for semilinear waves with two dissipative terms
}

\author{
Wenhui Chen ${ }^{1} \cdot$ Marcello D'Abbicco $^{2} \cdot$ Giovanni Girardi $^{2}$ iD
}

Received: 3 October 2020 / Accepted: 4 June 2021 / Published online: 18 June 2021

(c) The Author(s) 2021

\begin{abstract}
In this work, we prove the existence of global (in time) small data solutions for wave equations with two dissipative terms and with power nonlinearity $|u|^{p}$ or nonlinearity of derivative type $\left|u_{t}\right|^{p}$, in any space dimension $n \geqslant 1$, for supercritical powers $p>\bar{p}$. The presence of two dissipative terms strongly influences the nature of the problem, allowing us to derive $L^{r}-L^{q}$ long time decay estimates for the solution in the full range $1 \leqslant r \leqslant q \leqslant \infty$. The optimality of the critical exponents is guaranteed by a nonexistence result for subcritical powers $p<\bar{p}$.
\end{abstract}

Keywords Semilinear wave equation $\cdot$ Structural damping $\cdot$ Critical exponent $\cdot$ Global in time existence $\cdot$ Blow-up

Mathematics Subject Classification Primary 35L15 - 35L71 - Secondary 35B33 - 35A01 . 35B44

\section{Introduction}

In this paper, we study the following Cauchy problem for semilinear wave equations with two dissipative terms:

$$
\begin{cases}u_{t t}-\Delta u+(-\Delta)^{\rho} u_{t}+(-\Delta)^{\theta} u_{t}=F\left(u, u_{t}\right), & x \in \mathbb{R}^{n}, t>0, \\ u(0, x)=0, u_{t}(0, x)=u_{1}(x), & x \in \mathbb{R}^{n},\end{cases}
$$

with $0 \leqslant \rho<1 / 2<\theta \leqslant 1$, where the nonlinearity $F=F\left(u, u_{t}\right)$ on the right-hand side can be described by

Giovanni Girardi

giovanni.girardi@uniba.it

Wenhui Chen

wenhui.chen.math@gmail.com

Marcello D’Abbicco

marcello.dabbicco@uniba.it

1 School of Mathematical Sciences, Shanghai Jiao Tong University, 200240 Shanghai, China

2 Department of Mathematics, University of Bari, Via E. Orabona 4, 70125 Bari, Italy 


$$
F\left(u, u_{t}\right)=\left|\partial_{t}^{j} u\right|^{p} \quad \text { with } p>1 \text {, and } j=0 \text { or } j=1 .
$$

The critical exponent for the Cauchy problem (1) with $F=|u|^{p}$ or $F=\left|u_{t}\right|^{p}$, is, respectively, given by $p_{0}(n, \rho)$ or $p_{1}(n, \rho)$, where

$$
\begin{gathered}
p_{0}(n, \rho)=1+\frac{2}{(n-2 \rho)^{+}}, \\
p_{1}(n, \rho)=1+\frac{2 \rho}{n} .
\end{gathered}
$$

If $p \in\left(1, p_{j}(n, \rho)\right]$ (subcritical and critical cases), it is easy to prove that problem (1) admits no global (in time) weak solution, for initial data verifying a suitable sign assumption (Proposition 2.1). If $p>p_{j}(n, \rho)$ (supercritical case), then we prove that there exists a unique global (in time) solution for sufficiently small data (Theorem 2.1 and Corollary 2.1) in an appropriate space. In Theorems 2.1 and 2.2 we prove the existence of global (in time) energy solutions $u \in \mathcal{C}\left([0, \infty), L^{\eta} \cap H^{2 \theta}\right) \cap \mathcal{C}^{1}\left([0, \infty), L^{2}\right)$ to (1) with $F=|u|^{p}$ where, respectively, $\eta=1$ or $\eta \in(1,2]$, assuming small initial data in $L^{\eta} \cap L^{2}$. We call them "energy solutions" since the energy functional

$$
\frac{1}{2}\left\|u_{t}(t, \cdot)\right\|_{L^{2}}^{2}+\frac{1}{2}\|\nabla u(t, \cdot)\|_{L^{2}}^{2}
$$

is well-defined and continuous. In Theorem 2.3, we do not consider energy solutions, but we prove the existence of global (in time) weak solutions $u \in \mathcal{C}^{1}\left([0, \infty), L^{\eta} \cap L^{\eta p}\right)$ to (1) with $F=\left|u_{t}\right|^{p}$, for some $\eta>1$, assuming small initial data in $L^{\eta} \cap L^{\eta p}$.

In the last years, dissipative wave equations attracted a lot of attention. Let us first consider the linear wave equation with friction and viscoelastic damping, namely

$$
\begin{cases}u_{t t}-\Delta u+u_{t}-\Delta u_{t}=0, & x \in \mathbb{R}^{n}, t>0, \\ u(0, x)=u_{0}(x), u_{t}(0, x)=u_{1}(x), & x \in \mathbb{R}^{n},\end{cases}
$$

and the corresponding nonlinear problem. Asymptotic profiles of solutions to (5) are derived in $[1,2]$. In [3], the second author showed that the presence of two damping terms in (5) allows to derive $L^{p}-L^{q}$ estimates in the full range $1 \leqslant p \leqslant q \leqslant \infty$, in any space dimension $n \geqslant 1$. These estimates may be effectively used to study global (in time) existence of small data solutions to

$$
\left\{\begin{array}{l}
u_{t t}-\Delta u+u_{t}-\Delta u_{t}=F\left(u, u_{t}\right), \quad x \in \mathbb{R}^{n}, t>0, \\
u(0, x)=u_{0}(x), u_{t}(0, x)=u_{1}(x), x \in \mathbb{R}^{n},
\end{array}\right.
$$

where $F=F\left(u, u_{t}\right)$ is the same as in (2). In particular, these estimates allow us to prove global (in time) existence of small data solutions for any $p>1$ in the case $F=\left|u_{t}\right|^{p}$. Other studies on doubly dissipative wave models can be found in $[4,5]$ and in the references therein.

The main advantage of the presence of two dissipative term in (5) may be understood noticing that the value $\sigma=1 / 2$ is a threshold between two different asymptotic profiles for the solution to the damped wave equation 


$$
\left\{\begin{array}{l}
u_{t t}-\Delta u+(-\Delta)^{\sigma} u_{t}=F\left(u, u_{t}\right), \quad x \in \mathbb{R}^{n}, t>0, \\
u(0, x)=u_{0}(x), u_{t}(0, x)=u_{1}(x), x \in \mathbb{R}^{n}
\end{array}\right.
$$

According to the classification introduced in [6], the case $\sigma \in[0,1 / 2)$ corresponds to an effective dissipation. In this case, the asymptotic profile and the critical exponent for (7) with $F=|u|^{p}$ are the same of the corresponding heat equation with suitable initial data if $\sigma=0$ [7-9]. A similar phenomenon appears when $\sigma \in(0,1 / 2)$ but in this case two possible asymptotic profiles of anomalous diffusion appear; one profile is dominant if $F=|u|^{p}$ (see [10-12]) and the other profile is dominant if $F=\left|u_{t}\right|^{p}$ (see [13]). On the other hand, the case $\sigma \in(1 / 2,1]$ corresponds to a noneffective dissipation: the asymptotic profile of the solution to (7) contains oscillations analogous as the undamped wave equation (see [14-19]). However, the noneffective dissipation produces a better smoothing effect than the effective one. This is due to the fact that the noneffective dissipation produces a parabolic smoothing effect; for instance, the problem is well-posed in $L^{p}$, for $p \in[1, \infty]$. On the other hand, the effective dissipation does not help to manage the regularity issues typical of the wave equation and other hyperbolic equations; for instance, the problem is well-posed in $H^{s}$ spaces, but not in $L^{p}$ spaces, when $p \neq 2$.

The threshold case $\sigma=1 / 2$ inherits the benefits of the both effective and noneffective dissipations, and this allows to obtain the global (in time) existence of small data solutions in any space dimension $n \geqslant 1$ in the critical case (see [20]). The benefits of the both effective and noneffective dissipations are also gained in model (5), but with several important differences. The model in (1) describes the transition between the two regimens. For this reason, we expect that the critical exponents are the same as those in the effective case, namely $p_{0}$ and $p_{1}$ in (3) and (4), but the smoothing typical of the noneffective dissipation allows us to prove our result for any space dimension $n \geqslant 1$.

To prove the desired global (in time) existence results, we first derive long time decay estimates for the solution to the linear Cauchy problem

$$
\begin{cases}u_{t t}-\Delta u+(-\Delta)^{\rho} u_{t}+(-\Delta)^{\theta} u_{t}=0, & x \in \mathbb{R}^{n}, t>0, \\ u(0, x)=0, u_{t}(0, x)=u_{1}(x), & x \in \mathbb{R}^{n},\end{cases}
$$

with $0 \leqslant \rho<1 / 2<\theta \leqslant 1$. We cannot directly follow the approach in [3] to derive the $L^{1}-L^{1}$ estimate for (5) at high frequencies. In order to overcome this difficulty, we expand the kernel of solutions in a suitable way and we apply the Mikhlin-Hörmander multiplier theorem and Hardy-Littlewood theorem for the Riesz potential, to get some $L^{r}-L^{q}$ estimates, with $1<r \leqslant q<\infty$.

This paper is organized as follows. In Sect. 2, we state our main results on global (in time) existence of small data solution and blow-up of solutions to (1). In Sect. 3, we prepare $L^{r}-L^{q}$ low frequencies estimates and $L^{m}-L^{q}$ high frequencies estimates for the linear Cauchy problem (8), where $1 \leqslant r \leqslant m \leqslant q \leqslant \infty$. Then, applying the derived estimates and Banach's fixed point theorem, we prove global (in time) existence results for (1) with $|u|^{p}$ or $\left|u_{t}\right|^{p}$, in Sect. 4. Eventually, in Sect. 5, the blow-up results in the subcritical case for (1) are derived.

\subsection{Notation}

We write $f \lesssim g$ when there exists a positive constant $C$ such that $f \leqslant C g$.

For any $x \in \mathbb{R}$, we define $(x)^{+}=\max \{x, 0\}$ and $1 /(x)^{+}=\infty$ when $x \leqslant 0$. We define $\lceil x\rceil^{+}=(\lceil x\rceil)^{+}$, where the ceiling function $\lceil x\rceil$ denotes the smallest integer larger than $x$, i.e., 


$$
\lceil x\rceil=\min \{m \in \mathbb{Z}: x \leqslant m\} .
$$

For $b \geqslant 0$, we define $(-\Delta)^{\frac{b}{2}} f=\mathcal{F}^{-1}\left(|\xi|^{b} \hat{f}\right)$, the fractional Laplace operator, and $I_{b} f=\mathcal{F}^{-1}\left(|\xi|^{-b} \hat{f}\right)$ the Riesz potential operator; $\hat{f}=\mathcal{F}(f)$ denotes the Fourier transform of a function $f$.

For any $q \in[1, \infty]$ and $m \in \mathbb{N}, W_{q}^{m}=\left\{f \in L^{q}: \partial_{x}^{\alpha} f \in L^{q},|\alpha| \leqslant m\right\}$ denotes the usual Sobolev space of order $m$. For $s \in(0, \infty)$, noninteger, and $q \in(1, \infty), W_{q}^{s}$ denotes the Bessel potential space $W_{q}^{s}=\left\{f \in L^{q}:(1-\Delta)^{s / 2} f \in L^{q}\right\}$.

\section{Main results}

We first consider the case of power nonlinearity $F=|u|^{p}$.

Theorem 2.1 Let $n \geqslant 1$ and $0 \leqslant \rho<1 / 2<\theta \leqslant 1$. Let us assume

$$
p>p_{0}(n, \rho)=1+\frac{2}{n-2 \rho},
$$

where $p_{0}$ is defined in (3). Also we assume that $p<n /(n-4 \theta)$ if $n>4 \theta$. Then, there exists a constant $\epsilon>0$ such that for any

$$
u_{1} \in L^{1} \cap L^{2} \text { with }\left\|u_{1}\right\|_{L^{1} \cap L^{2}} \leqslant \epsilon,
$$

there is a uniquely determined energy solution

$$
u \in \mathcal{C}\left([0, \infty), L^{1} \cap H^{2 \theta}\right) \cap \mathcal{C}^{1}\left([0, \infty), L^{2}\right)
$$

to (1) with $F\left(u, u_{t}\right)=|u|^{p}$. Furthermore, the solution satisfies the following estimates:

$$
\begin{gathered}
\left\|(-\Delta)^{\theta} u(t, \cdot)\right\|_{L^{2}} \lesssim(1+t)^{-\frac{n}{4(1-\rho)}-\frac{\theta-\rho}{1-\rho}}\left\|u_{1}\right\|_{L^{1} \cap L^{2}}, \\
\|u(t, \cdot)\|_{L^{\bar{q}}} \lesssim\left\|u_{1}\right\|_{L^{1} \cap L^{2}}, \\
\|u(t, \cdot)\|_{L^{1}} \lesssim(1+t)\left\|u_{1}\right\|_{L^{1} \cap L^{2}},
\end{gathered}
$$

where $\bar{q}=n /(n-2 \rho)$, and

$$
\left\|u_{t}(t, \cdot)\right\|_{L^{2}} \lesssim \begin{cases}(1+t)^{-\frac{1}{4 \rho}}\left\|u_{1}\right\|_{L^{1} \cap L^{2}} & \text { if } n=1 \text { and } \rho \in(1 / 4,1 / 2), \\ (1+t)^{-\frac{n}{4(1-\rho)}-\frac{1-2 \rho}{1-\rho}}\left\|u_{1}\right\|_{L^{1} \cap L^{2}} & \text { otherwise. }\end{cases}
$$

If we replace the smallness of initial data in $L^{1}$ by the smallness of initial data in $L^{\eta}$, $\eta>1$, the critical exponent becomes $p_{0}(n / \eta, \rho)$, as discussed in [13]. We demonstrate the global (in time) existence of energy solutions with small data in $L^{\eta}$, for supercritical and critical powers $p \geqslant p_{0}(n / \eta, \rho)$.

Theorem 2.2 Let $n \geqslant 1$ and $0 \leqslant \rho<1 / 2<\theta \leqslant 1$. Let us fix $\eta \in(1,2]$, such that $2 \rho \eta<1$ if $n=1$, and assume 


$$
p \geqslant p_{0}(n / \eta, \rho)=1+\frac{2 \eta}{n-2 \rho \eta},
$$

where $p_{0}$ is defined in (3). Also we assume that $p<n /(n-4 \theta)$ if $n>4 \theta$. Then, there exists a constant $\epsilon>0$ such that for any

$$
u_{1} \in L^{\eta} \cap L^{2} \text { with }\left\|u_{1}\right\|_{L^{\eta} \cap L^{2}} \leqslant \epsilon,
$$

there is a uniquely determined energy solution

$$
u \in \mathcal{C}\left([0, \infty), L^{\eta} \cap H^{2 \theta}\right) \cap \mathcal{C}^{1}\left([0, \infty), L^{2}\right)
$$

to (1) with $F=|u|^{p}$. Furthermore, the solution satisfies the following estimates:

$$
\begin{gathered}
\left\|(-\Delta)^{\theta} u(t, \cdot)\right\|_{L^{2}} \\
\lesssim \begin{cases}(1+t)^{-\frac{n}{2(1-\rho)}\left(\frac{1}{\eta}-\frac{1}{2}\right)-\frac{\theta-\rho}{1-\rho}}\left\|u_{1}\right\|_{L^{\eta} \cap L^{2}} & \text { if } p>p_{0}(n / \eta, \rho) \text { or } \theta<1, \\
(1+t)^{-\frac{n}{2(1-\rho)}\left(\frac{1}{\eta}-\frac{1}{2}\right)-1} \log (2+t)\left\|u_{1}\right\|_{L^{\eta} \cap L^{2}} & \text { if } p=p_{0}(n / \eta, \rho) \text { and } \theta=1,\end{cases} \\
\|u(t, \cdot)\|_{L^{\bar{q}}} \lesssim\left\|u_{1}\right\|_{L^{\eta} \cap L^{2}}, \\
\|u(t, \cdot)\|_{L^{\eta}} \lesssim(1+t)\left\|u_{1}\right\|_{L^{\eta} \cap L^{2}},
\end{gathered}
$$

where $\bar{q}=n \eta /(n-2 \rho \eta)$, and

$$
\left\|u_{t}(t, \cdot)\right\|_{L^{2}} \lesssim \begin{cases}(1+t)^{-\frac{n}{2(1-\rho)}\left(\frac{1}{\eta}-\frac{1}{2}\right)-\frac{1-2 \rho}{1-\rho}}\left\|u_{1}\right\|_{L^{1} \cap L^{2}} & \text { if } \bar{q}<2, \\ (1+t)^{-\frac{1}{2 \rho}\left(\frac{1}{\eta}-\frac{1}{2}\right)}\left\|u_{1}\right\|_{L^{1} \cap L^{2}} & \text { if } \bar{q} \geqslant 2 \text { and } p>p_{0}(n / \eta, \rho), \\ (1+t)^{-\frac{1}{2 \rho}\left(\frac{1}{\eta}-\frac{1}{2}\right)} \log (2+t)\left\|u_{1}\right\|_{L^{1} \cap L^{2}} & \text { if } \bar{q} \geqslant 2 \text { and } p=p_{0}(n / \eta, \rho) .\end{cases}
$$

Remark 2.1 Using Gagliardo-Nirenberg inequality, as a consequence of (12) and (13) in Theorem 2.1 or, respectively, (18) and (19) in Theorem 2.2, the solution to (1) with $F=|u|^{p}$ verifies the decay estimate

$$
\|u(t, \cdot)\|_{L^{q}} \lesssim(1+t)^{-\frac{n}{2(1-\rho)}\left(\frac{1}{\eta}-\frac{1}{q}\right)+\frac{\rho}{1-\rho}}\left\|u_{1}\right\|_{L^{\eta} \cap L^{2}}
$$

for any $q \in(\bar{q}, \infty]$ if $n<4 \theta$, for any $q \in(\bar{q}, \infty)$ if $n=4 \theta$, and for any $q \in(\bar{q}, 2 n /(n-4 \theta)]$ if $n>4 \theta$ (exception given for the case $p=p_{0}(n / \eta, \rho)$ and $\left.\theta=1\right)$. On the other hand, interpolating (13) and (14) in Theorem 2.1 or, respectively, (19) and (20) in Theorem 2.2, the solution to (1) with $F=|u|^{p}$ verifies the estimate

$$
\|u(t, \cdot)\|_{L^{q}} \lesssim(1+t)^{-\frac{n}{2 \rho}\left(\frac{1}{\eta}-\frac{1}{q}\right)+1}\left\|u_{1}\right\|_{L^{\eta} \cap L^{2}},
$$

for any $q \in[\eta, \bar{q}]$. The latter is not a decay estimate. We mention that in the both cases, the estimate is the same of the solution to the linear problem (8), whose optimality is guaranteed by the diffusion phenomenon [10]. 
Remark 2.2 Actually, one may apply Theorem 2.2 to obtain the global (in time) existence result for (1) with $F=|u|^{p}$ and small initial data in $L^{1} \cap L^{2}$ for any $p>p_{0}(n, \rho)$. Indeed, for a given $p>p_{0}(n, \rho)$, it is sufficient to apply Theorem 2.2 with some $\eta \in(1,2]$ such that $p \geqslant p_{0}(n / \eta, \rho)$. This strategy will be used to deal with (1) with $F=\left|u_{t}\right|^{p}$, to avoid the more challenging $L^{1}-L^{1}$ estimates for $u_{t}$. This difficulty is one peculiar difference with respect to the case $(\rho, \theta)=(0,1)$ studied in [3].

Theorem 2.3 Let $n \geqslant 1$ and $0 \leqslant \rho<1 / 2<\theta \leqslant 1$. Let us fix $\eta \in(1, \infty)$ and assume

$$
p \geqslant p_{1}(n / \eta, \rho)=1+\frac{2 \rho \eta}{n},
$$

where $p_{1}$ is defined in (4). Also we assume that $p \leqslant n /(n-2 \rho \eta)$ if $n \geqslant 2$, or if $n=1$ and $2 \rho \eta<1$. Then, there exists $\epsilon>0$ such that for any

$$
u_{1} \in L^{\eta} \cap L^{\eta p} \quad \text { with } \quad\left\|u_{1}\right\|_{L^{\eta} \cap L^{\eta p}} \leqslant \epsilon,
$$

there is a uniquely determined weak solution

$$
u \in \mathcal{C}^{1}\left([0, \infty), L^{\eta} \cap L^{\eta p}\right)
$$

to (1) with $F=\left|u_{t}\right|^{p}$. Furthermore, the solution satisfies the following estimates:

$$
\begin{gathered}
\left\|\partial_{t}^{j} u(t, \cdot)\right\|_{L^{\eta}} \lesssim(1+t)^{1-j}\left\|u_{1}\right\|_{L^{\eta} \cap L^{\eta p}}, \\
\left\|\partial_{t}^{j} u(t, \cdot)\right\|_{L^{\eta p}} \lesssim(1+t)^{-\frac{n}{2 \rho \eta}\left(1-\frac{1}{p}\right)+1-j}\left\|u_{1}\right\|_{L^{\eta} \cap L^{\eta p}},
\end{gathered}
$$

for $j=0,1$.

Thanks to Theorem 2.3, we immediately obtain the following.

Corollary 2.1 Let $n \geqslant 1$ and $0 \leqslant \rho<1 / 2<\theta \leqslant 1$. Let us assume

$$
p>p_{1}(n, \rho)=1+\frac{2 \rho}{n},
$$

where $p_{1}$ is defined in (4). We also assume that $p \leqslant n /(n-2 \rho)$. Let us fix $q \in(p, \infty)$. Then, there exists $\epsilon>0$ such that for any

$$
u_{1} \in L^{1} \cap L^{q} \quad \text { with } \quad\left\|u_{1}\right\|_{L^{1} \cap L^{q}} \leqslant \epsilon,
$$

and for any $\eta \in(1, q / p]$ such that $p \geqslant p_{1}(n / \eta, \rho)$, there is a uniquely determined weak solution

$$
u \in \mathcal{C}^{1}\left([0, \infty), L^{\eta} \cap L^{\eta p}\right)
$$

to (1) with $F=\left|u_{t}\right|^{p}$. Furthermore, the solution satisfies the estimates (24) and (25), for $j=0,1$. 
Remark 2.3 If $\eta p \geqslant 2$, or if the initial datum is assumed to be small also in $L^{2}$, it is not difficult to modify the proof of Theorem 2.3 or Corollary 2.1 , and construct an energy solution in $\mathcal{C}\left([0, \infty), H^{2 \theta}\right) \cap \mathcal{C}^{1}\left([0, \infty), L^{\eta} \cap L^{\eta p}\right)$.

The optimality of the critical exponents $p_{j}(n / \eta, \rho)$ in Theorems 2.1, 2.2, 2.3 and in Corollary 2.1 is guaranteed by the counterpart of nonexistence results in the subcritical and critical cases $p \in\left(1, p_{j}(n, \rho)\right]$, if $\eta=1$, and in the subcritical case $p \in\left(1, p_{j}(n / \eta, \rho)\right)$, if $\eta>1$. Even if these results are easily derived following as in $[13,21,22]$, using the test function method [23-28], we will sketch the outlines of the statement and its proof.

Proposition 2.1 Let $n \geqslant 1$ and $0 \leqslant \rho<1 / 2<\theta \leqslant 1$. Let us assume that $u_{1} \in L^{\eta}$ with $\eta \geqslant 1$ verifies the following sign condition:

$$
\begin{gathered}
\int_{\mathbb{R}^{n}} u_{1}(x) \mathrm{d} x>0 \quad \text { if } u_{1} \in L^{1}, \\
u_{1}(x) \geqslant \epsilon|x|^{-\frac{n}{n}}(\log (1+|x|))^{-1} \quad \text { for }|x| \geqslant 1 \text { if } u_{1} \in L^{\eta} \text { with } \eta>1,
\end{gathered}
$$

where $\epsilon>0$. For $j=0,1$, fix $p \in\left(1, p_{j}(n, \rho)\right]$ if $\eta=1$ or $p \in\left(1, p_{j}(n / \eta, \rho)\right)$ if $\eta>1$. Then, there is no (weak) global (in time) solution to (1) with $F=\left|\partial_{t}^{j} u\right|^{p}$.

\section{Estimates of solutions to the linear problem}

Let us apply the partial Fourier transform with respect to $x$ to (8), defining $\hat{u}(t, \xi)=\mathcal{F}_{x \rightarrow \xi}(u(t, x))$. Then, we obtain the initial value problem

$$
\begin{cases}\hat{u}_{t t}+\left(|\xi|^{2 \rho}+|\xi|^{2 \theta}\right) \hat{u}_{t}+|\xi|^{2} \hat{u}=0, & \xi \in \mathbb{R}^{n}, t>0, \\ \hat{u}(0, \xi)=0, \hat{u}_{t}(0, \xi)=\hat{u}_{1}(\xi), & \xi \in \mathbb{R}^{n}\end{cases}
$$

The roots of the characteristic equation

$$
\lambda^{2}+\left(|\xi|^{2 \rho}+|\xi|^{2 \theta}\right) \lambda+|\xi|^{2}=0
$$

can be expressed by

$$
\lambda_{ \pm}(|\xi|)= \begin{cases}-\frac{1}{2}\left(\left(|\xi|^{2 \rho}+|\xi|^{2 \theta}\right) \pm \sqrt{\left(|\xi|^{2 \rho}+|\xi|^{2 \theta}\right)^{2}-4|\xi|^{2}}\right) & \text { if } \xi \in \mathbb{R}^{n} \backslash \Omega_{\rho, \theta}, \\ -\frac{1}{2}\left(\left(|\xi|^{2 \rho}+|\xi|^{2 \theta}\right) \pm i \sqrt{4|\xi|^{2}-\left(|\xi|^{2 \rho}+|\xi|^{2 \theta}\right)^{2}}\right) & \text { if } \quad \xi \in \Omega_{\rho, \theta},\end{cases}
$$

where $\Omega_{\rho, \theta}=\left\{\xi \in \mathbb{R}^{n}:|\xi|^{2 \rho-1}+|\xi|^{2 \theta-1}<2\right\}$.

Definition 3.1 We introduce three cut-off functions, namely $\chi_{\text {int }}(\xi), \chi_{\text {mid }}(\xi)$ and $\chi_{\text {ext }}(\xi) \in \mathcal{C}^{\infty}\left(\mathbb{R}^{n}\right)$ supported, respectively, in 


$$
\begin{aligned}
\mathcal{Z}_{\text {int }}(\varepsilon) & =\left\{\xi \in \mathbb{R}^{n}:|\xi| \leqslant \varepsilon\right\}, \\
\mathcal{Z}_{\text {mid }}(\varepsilon, N) & =\left\{\xi \in \mathbb{R}^{n}: \varepsilon / 2 \leqslant|\xi| \leqslant 2 N\right\}, \\
\mathcal{Z}_{\text {ext }}(N) & =\left\{\xi \in \mathbb{R}^{n}:|\xi| \geqslant N\right\},
\end{aligned}
$$

for a sufficiently small $\varepsilon>0$ and a sufficiently large $N \gg 1$, and such that $\chi_{\text {int }}(\xi)+\chi_{\text {mid }}(\xi)+\chi_{\text {ext }}(\xi)=1$.

In particular, we fix $\varepsilon, N$ so that $\Omega_{\rho, \theta} \subset \mathcal{Z}_{\text {mid }}(3 \varepsilon, N / 3)$.

Here and hereafter

$$
\begin{aligned}
\chi_{\text {int }}(D) u(t, x) & =\mathcal{F}^{-1}\left[\chi_{\text {int }}(\xi) \hat{u}(t, \cdot)\right](x), \\
\chi_{\text {mid }}(D) u(t, x) & =\mathcal{F}^{-1}\left[\chi_{\text {mid }}(\xi) \hat{u}(t, \cdot)\right](x), \\
\chi_{\text {ext }}(D) u(t, x) & =\mathcal{F}^{-1}\left[\chi_{\text {ext }}(\xi) \hat{u}(t, \cdot)\right](x) .
\end{aligned}
$$

The solution to (8) localized in $\mathcal{Z}_{\text {mid }}(\varepsilon, N)$ verifies the estimate

$$
\left\|\partial_{t}^{j} \chi_{\text {mid }}(D) u(t, \cdot)\right\|_{W^{2 s, r},} \leqslant C \mathrm{e}^{-c t}\left\|u_{1}\right\|_{L^{q}},
$$

for any $1 \leqslant r \leqslant q \leqslant \infty, j+s \geqslant 0$, and $t \geqslant 0$, for some $C, c>0$, independent of the datum. Indeed, $\chi_{\text {mid }}$ is compactly supported in $\mathbb{R}^{n} \backslash\{0\}$ and the real parts of the roots $\lambda_{ \pm}(\xi)$ are negative in $\mathbb{R}^{n} \backslash\{0\}$. Thus, it will be sufficient to estimate the solution at low and high frequencies, that is, we will estimate $\chi_{\text {int }}(D) u$ and $\chi_{\text {ext }}(D) u$.

Let $K_{1}=K_{1}(t,|x|)$ denote the fundamental solution to (8), that is,

$$
u(t, x)=K_{1}(t,|x|) *_{(x)} u_{1}(x) .
$$

Then, it holds

$$
\left(\mathcal{F}_{x \rightarrow \xi} K_{1}\right)(t,|\xi|)=\frac{\mathrm{e}^{\lambda_{-}(|\xi|) t}-\mathrm{e}^{\lambda_{+}(|\xi|) t}}{\lambda_{-}(|\xi|)-\lambda_{+}(|\xi|)}, \quad \forall \xi \in \mathcal{Z}_{\text {int }}(\varepsilon) \cup \mathcal{Z}_{\text {ext }}(N) .
$$

If we define

$$
\gamma(|\xi|)=\sqrt{1-4|\xi|^{2}\left(|\xi|^{2 \rho}+|\xi|^{2 \theta}\right)^{-2}},
$$

then the roots and their difference can be expressed by

$$
\begin{gathered}
\lambda_{+}(|\xi|)=-\frac{1}{2}\left(|\xi|^{2 \rho}+|\xi|^{2 \theta}\right)(1+\gamma(|\xi|)) \\
\lambda_{-}(|\xi|)=-\frac{1}{2}\left(|\xi|^{2 \rho}+|\xi|^{2 \theta}\right)(1-\gamma(|\xi|))=-\frac{2|\xi|^{2}}{\left(|\xi|^{2 \rho}+|\xi|^{2 \theta}\right)(1+\gamma(|\xi|))} \\
\lambda_{-}(|\xi|)-\lambda_{+}(|\xi|)=\left(|\xi|^{2 \rho}+|\xi|^{2 \theta}\right) \gamma(|\xi|) .
\end{gathered}
$$

To obtain the desired estimates at low frequencies, we rely on the following lemma.

Lemma 3.1 (Lemma 3.1 in [10]). Let us consider 


$$
K(t, x) *_{(x)} h=\mathcal{F}_{\xi \rightarrow x}^{-1}\left(f(|\xi|) \mathrm{e}^{-g(|\xi|) t} \hat{h}(\xi)\right),
$$

where $f$ and $g$ satisfy the following conditions:

$$
\left|\partial_{z}^{k} f(z)\right| \lesssim z^{\alpha-k}, \quad\left|\partial_{z}^{k} g(z)\right| \lesssim z^{-k} g(z) \quad \text { and } \quad b_{0} z^{\beta} \leqslant g(z) \leqslant b_{1} z^{\beta}
$$

for some $b_{0}>0$ and $\alpha>-1, \beta>0$, real-valued, for any $z \in(0, \varepsilon)$ with $\varepsilon \ll 1$ and $k \leqslant[(n+3) / 2]$, which denotes the integer part of $(n+3) / 2$. Then we have the following $L^{r}-L^{q}$ estimates:

$$
\left\|\chi_{\text {int }}(D) K(t, x) *_{(x)} h\right\|_{L^{q}} \lesssim(1+t)^{-\frac{n}{\beta}\left(\frac{1}{r}-\frac{1}{q}\right)-\frac{\alpha}{\beta}}\left\|\chi_{\text {int }}(D) h\right\|_{L^{r}}
$$

in the following cases:

- For any $1 \leqslant r \leqslant q \leqslant \infty$, if $\alpha>0$ or if $f(z)=A$ is a nonzero constant;

- For any $1 \leqslant r<q \leqslant \infty$, if $\alpha=0$ but $f$ is not a constant;

- For any pair $(r, q)$ such that $1 / r-1 / q \geqslant-\alpha / n$, if $\alpha \in(-1,0)$.

Applying Lemma 3.1, the following $L^{r}-L^{q}$ estimates follow.

Proposition 3.1 Let $u$ be the solution to (8) with $0 \leqslant \rho<1 / 2<\theta \leqslant 1$. Then, for any $1 \leqslant r \leqslant q \leqslant \infty, s \geqslant 0$, and for $j=0,1$, we have

$$
\begin{aligned}
\left\|\chi_{\text {int }}(D)(-\Delta)^{s} \partial_{t}^{j} u(t, \cdot)\right\|_{L^{q}} \\
\quad \lesssim(1+t)^{\max \left\{-\frac{n}{2(1-\rho)}\left(\frac{1}{r}-\frac{1}{q}\right)+\frac{\rho-s}{1-\rho},-\frac{n}{2 \rho}\left(\frac{1}{r}-\frac{1}{q}\right)+\frac{\rho-s}{\rho}\right\}-j}\left\|u_{1}\right\|_{L^{r}} \\
\quad=\left\{\begin{array}{lll}
(1+t)^{-\frac{n}{2(1-\rho)}\left(\frac{1}{r}-\frac{1}{q}\right)+\frac{\rho-s}{1-\rho}-j}\left\|u_{1}\right\|_{L^{r}} & \text { if } & \frac{1}{r}-\frac{1}{q} \geqslant \frac{2(\rho-s)}{n}, \\
(1+t)^{-\frac{n}{2 \rho}\left(\frac{1}{r}-\frac{1}{q}\right)+1-j-\frac{s}{\rho}}\left\|u_{1}\right\|_{L^{r}} & \text { if } & \frac{1}{r}-\frac{1}{q} \leqslant \frac{2(\rho-s)}{n} .
\end{array}\right.
\end{aligned}
$$

Remark 3.1 As expected, estimates in Proposition 3.1 are consistent with the low frequencies estimate obtained in [10, Corollary 2.2] for the solution to (7) with $F=0$ and $\sigma=\rho$. The optimality of these estimates is guaranteed by the diffusion phenomenon: the solution localized at low frequencies may be decomposed in two terms. Each term asymptotically behaves as the solution to an anomalous diffusion problem, namely

$$
\mathrm{e}^{-(-\Delta)^{1-\rho} t} I_{2 \rho} u_{1} \text { and } \mathrm{e}^{-(-\Delta)^{\rho} t} I_{2 \rho} u_{1}
$$

where $I_{2 \rho}$ denotes the Riesz potential.

Proof We assume $\rho>0$, since the case $\rho=0$ is easier; indeed, in this latter case, the term $\mathrm{e}^{\lambda_{+}(|\xi|) t}$ produces an exponential decay.

First let us consider $j=1$. In view of representation (31), we may apply Lemma 3.1, with $g_{ \pm}(|\xi|)=-\lambda_{ \pm}(|\xi|)$ and with 


$$
f_{ \pm}(|\xi|)=-\frac{|\xi|^{2 s} \lambda_{ \pm}(|\xi|)}{\lambda_{-}(|\xi|)-\lambda_{+}(|\xi|)}
$$

It is convenient to write

$$
\begin{aligned}
& f_{+}(|\xi|)=\frac{|\xi|^{2 s}(1+\gamma(|\xi|))}{2 \gamma(|\xi|)} \\
& f_{-}(|\xi|)=\frac{2|\xi|^{2 s+2}}{\left(|\xi|^{2 \rho}+|\xi|^{2 \theta}\right)^{2}(1+\gamma(|\xi|)) \gamma(|\xi|)} .
\end{aligned}
$$

By straightforward computation, the roots $\lambda_{ \pm}(|\xi|)$ verify the estimates

$$
\begin{aligned}
\left|\partial_{|\xi|}^{k}\left(-\lambda_{+}(|\xi|)\right)\right| & \lesssim|\xi|^{2 \rho-k} \lesssim|\xi|^{-k}\left(-\lambda_{+}(|\xi|)\right), \\
\left|\partial_{|\xi|}^{k}\left(-\lambda_{-}(|\xi|)\right)\right| & \lesssim|\xi|^{2-2 \rho-k} \lesssim|\xi|^{-k}\left(-\lambda_{-}(|\xi|)\right), \\
b_{1}|\xi|^{2 \rho} & \leqslant-\lambda_{+}(|\xi|) \leqslant b_{2}|\xi|^{2 \rho} \\
b_{3}|\xi|^{2-2 \rho} & \leqslant-\lambda_{-}(|\xi|) \leqslant b_{4}|\xi|^{2-2 \rho}
\end{aligned}
$$

for some $b_{j}>0$, whereas

$$
\begin{aligned}
\left|\partial_{|\xi|}^{k} f_{+}(|\xi|)\right| & \lesssim|\xi|^{2 s-k} \\
\left|\partial_{|\xi|}^{k} f_{-}(|\xi|)\right| & \lesssim|\xi|^{2 s+2-4 \rho-k} .
\end{aligned}
$$

The above estimates are easily derived, due to the fact that neither $\lambda_{ \pm}$nor $f_{ \pm}$contain any oscillations, and that we are away from the zeroes of the root in $\gamma(|\xi|)$, due to the fact that $|\xi| \leqslant \varepsilon$. Namely, the estimates for the derivatives obey to the same rules of polynomials: each partial derivative with respect to $\xi$ produces a negative power of $|\xi|$. This property is essential in the framework of the theory of $L^{q}$ bounded operators, see for instance, Mikhlin-Hörmander multiplier theorem.

The desired estimate follows applying Lemma 3.1 with $\alpha=2 s, 2 s+2-4 \rho$, exception given for the term related to $f_{+}(|\xi|) \mathrm{e}^{\lambda_{+}(|\xi|) t}$ when $s=0$. In this latter case, we write

$$
f_{+}(|\xi|)=1+\frac{1-\gamma(|\xi|)}{2 \gamma(|\xi|)}=1+\frac{2|\xi|^{2}}{\left(|\xi|^{2 \rho}+|\xi|^{2 \theta}\right)^{2}(1+\gamma(|\xi|)) \gamma(|\xi|)}
$$

and we separately apply Lemma 3.1 with $\alpha=0$ and $A=1$, and with $\alpha=2-4 \rho$, to the two terms in the sum.

Now let us turn to $j=0$. The proof follows by integration, if $n(1 / r-1 / q)<2(\rho-s)$. Providing that $n(1 / r-1 / q) \geqslant 2(\rho-s)$, we set

$$
f(|\xi|)=\frac{|\xi|^{2 s}}{\lambda_{-}(|\xi|)-\lambda_{+}(|\xi|)}=\frac{|\xi|^{2 s}}{\left(|\xi|^{2 \rho}+|\xi|^{2 \theta}\right) \gamma(|\xi|)} .
$$

Due to the fact that

$$
\left|\partial_{|\xi|}^{k} f(|\xi|)\right| \lesssim|\xi|^{2 s-2 \rho-k}
$$


the proof follows, applying Lemma 3.1 with $\alpha=2 s-2 \rho$, exception given for the case $s=\rho$. In this latter case, we write

$$
f(|\xi|)=1-\frac{|\xi|^{2 \theta}}{|\xi|^{2 \rho}+|\xi|^{2 \theta}}+\frac{|\xi|^{2 \rho}(1-\gamma(|\xi|))}{\left(|\xi|^{2 \rho}+|\xi|^{2 \theta}\right) \gamma(|\xi|)},
$$

and we separately apply Lemma 3.1 with $\alpha=0$ and $A=1$, and with $\alpha=2 \theta-2 \rho$ and $\alpha=2-4 \rho$, to the three terms in the sum.

\section{1 $L^{m}-L^{q}$ estimates for high frequencies}

At high frequencies, we write

$$
\chi_{\text {ext }}(D) u(t, x)=\left(M_{-} u_{1}\right)(t, x)-\left(M_{+} u_{1}\right)(t, x),
$$

where

$$
\left(M_{ \pm} v\right)(t, x)=\mathcal{F}_{\xi \rightarrow x}^{-1}\left(\frac{\mathrm{e}^{\lambda_{ \pm}(|\xi|) t}}{\lambda_{-}(|\xi|)-\lambda_{+}(|\xi|)} \chi_{\text {ext }}(\xi) \hat{v}(\xi)\right)(t, x) .
$$

To estimate the derivatives with respect to $\xi$, we may proceed as we did at low frequencies in the proof of Proposition 3.1. Indeed, neither $\lambda_{ \pm}$nor $f_{ \pm}$contain any oscillations, and we are away from the zeroes of the root in $\gamma(|\xi|)$, due to the fact that $|\xi| \geqslant N$. For any $\beta \in \mathbb{N}^{n}$, we have the following estimates:

$$
\begin{aligned}
& \left|\partial_{\xi}^{\beta} \lambda_{+}(|\xi|)\right| \lesssim|\xi|^{2 \theta-|\beta|}, \quad\left|\partial_{\xi}^{\beta} \lambda_{-}(|\xi|)\right| \lesssim|\xi|^{2-2 \theta-|\beta|} \\
& \left|\lambda_{+}(|\xi|)-\lambda_{-}(|\xi|)\right| \approx|\xi|^{2 \theta} .
\end{aligned}
$$

Thanks to (36), we may easily derive $L^{q}-L^{q}$ estimates, for $q \in(1, \infty)$, using Mikhlin-Hörmander multiplier theorem.

Proposition 3.2 Let $n \geqslant 1, s \geqslant 0$ and $j \in \mathbb{N}$. Let $\kappa \geqslant 0$ and $q \in(1, \infty)$. Then, the solution to (8) localized at high frequencies verifies the estimates

$$
\left\|\partial_{t}^{j}(-\Delta)^{s} \chi_{\mathrm{ext}}(D) u(t, \cdot)\right\|_{L^{q}} \lesssim \mathrm{e}^{-c t} t^{-\kappa}\left\|u_{1}\right\|_{W_{q}^{[2 s+2 \theta(j-\kappa-1)]^{+}},}
$$

for any $t>0$ and positive constants $c$. Here, $\lceil x\rceil^{+}$denotes the positive part of the ceiling function of $x$, which was defined in (9).

Proof To prove our result, it is sufficient to show that

$$
\begin{gathered}
\left\|\partial_{t}^{j}(-\Delta)^{s}\left(M_{+} u_{1}\right)(t, \cdot)\right\|_{L^{q}} \lesssim \mathrm{e}^{-c t} t^{-\kappa}\left\|u_{1}\right\|_{W_{q}^{[2 s+2 \theta(j-\kappa-1)]^{+}},} \\
\left\|\partial_{t}^{j}(-\Delta)^{s}\left(M_{-} u_{1}\right)(t, \cdot)\right\|_{L^{q}} \lesssim \mathrm{e}^{-c t} t^{-\kappa}\left\|u_{1}\right\|_{W_{q}^{[2(1-\theta)(j-\kappa+1)+2 s-2]^{+}},}
\end{gathered}
$$

for $q \in(1, \infty)$ and for $\kappa \geqslant 0$ (we notice that the order of the Sobolev space in (39) is independent of $j, \kappa$ if $\theta=1)$. We define the multiplier 


$$
\hat{a}_{j, \kappa, s}(t, \xi)=\frac{\mathrm{e}^{\lambda_{+}(|\xi|) t} \lambda_{+}(|\xi|)^{j}|\xi|^{2 s}}{\left(\lambda_{-}(|\xi|)-\lambda_{+}(|\xi|)\right)\left(1+|\xi|^{2}\right)^{\lceil 2 s+2 \theta(j-\kappa-1)\rceil^{+} / 2}} \chi_{\text {ext }}(\xi) .
$$

So, the next representation holds:

$$
\partial_{t}^{j}(-\Delta)^{s}\left(M_{+} u_{1}\right)(t, x)=a_{j, \kappa, s}(t, x) *_{(x)}(1-\Delta)^{[2 s+2 \theta(j-\kappa-1)] / 2} u_{1}(x),
$$

for $2 s+2 \theta(j-\kappa-1) \geqslant 0$, where $a_{j, \kappa, s}=\mathcal{F}_{\xi \rightarrow x}^{-1}\left(\hat{a}_{j, \kappa, s}(t, \xi)\right)$.

In particular, we notice that

$$
\left|\partial_{\xi}^{\beta} \hat{a}_{j, \kappa, s}(t, \xi)\right| \lesssim \mathrm{e}^{-c t} t^{-\kappa}|\xi|^{-|\beta|} .
$$

In an analogous way, we define

$$
\hat{b}_{j, \kappa, s}(t, \xi)=\frac{\mathrm{e}^{\lambda_{-}(|\xi|) t} \lambda_{-}(|\xi|)^{j}|\xi|^{2 s}}{\left(\lambda_{-}(|\xi|)-\lambda_{+}(|\xi|)\right)\left(1+|\xi|^{2}\right)^{\left[2(1-\theta)(j-\kappa+1)-2+\left.2 s\right|^{+} / 2\right.}} \chi_{\text {ext }}(\xi),
$$

and we may represent

$$
\partial_{t}^{j}(-\Delta)^{s}\left(M_{-} u_{1}\right)(t, x)=b_{j, \kappa, s}(t, x) *_{(x)}(1-\Delta)^{\lceil 2(1-\theta)(j-\kappa+1)-2+2 s] / 2} u_{1}(x),
$$

for $2(1-\theta)(j-\kappa+1)-2+2 s \geqslant 0$, where $b_{j, \kappa, s}=\mathcal{F}_{\xi \rightarrow x}^{-1}\left(\hat{b}_{j, \kappa, s}(t, \xi)\right)$.

In this case, we may conclude

$$
\left|\partial_{\xi}^{\beta} \hat{b}_{j, K, s}(t, \xi)\right| \lesssim \mathrm{e}^{-c t} t^{-\kappa}|\xi|^{-|\beta|} .
$$

We complete the proof of (38-39), applying Mikhlin-Hörmander multiplier theorem.

Using Hardy-Littlewood-Sobolev theorem, we may extend Proposition 3.2 to $L^{m}-L^{q}$ estimates with $1<m<q<\infty$.

Proposition 3.3 Let $n \geqslant 1, s \geqslant 0$ and $j \in \mathbb{N}$. Let $\kappa \geqslant 0$ and $1<m<q<\infty$. Then, the solution to (8) localized at high frequencies verifies the estimates

$$
\left\|\partial_{t}^{j}(-\Delta)^{s} \chi_{\mathrm{ext}}(D) u(t, \cdot)\right\|_{L^{q}} \lesssim \mathrm{e}^{-c t} t^{-\kappa}\left\|u_{1}\right\|_{W_{m}^{[2 s+2 \theta(j-\kappa-1)+n(1 / m-1 / q)]^{+}},}
$$

for any $t>0$ and positive constants $c$.

Proof Let $\omega>0$ be defined by $1 / m-1 / q=\omega / n$. Then

$$
\begin{aligned}
\left\|\partial_{t}^{j}(-\Delta)^{s} \chi_{\mathrm{ext}}(D) K_{1}(t,|x|) *_{(x)} u_{1}\right\|_{L^{q}} \\
\quad=\left\|\partial_{t}^{j} \mathcal{F}_{\xi \rightarrow x}^{-1}\left(\chi_{\mathrm{ext}}(\xi)|\xi|^{2 s} \widehat{K_{1}}(t,|\xi|) \hat{u}_{1}(\xi)\right)\right\|_{L^{q}} \\
\quad=\left\|\partial_{t}^{j} \mathcal{F}_{\xi \rightarrow x}^{-1}\left(\chi_{\mathrm{ext}}(\xi) \widehat{K_{1}}(t,|\xi|)|\xi|^{2 s+\omega}\right) *_{(x)} I_{\omega} u_{1}\right\|_{L^{q}},
\end{aligned}
$$

where $I_{\omega}$ is the Riesz potential. Applying Theorem 3.2 with $s+\omega / 2$ in place of $s$, we can derive 


$$
\begin{gathered}
\left\|\partial_{t}^{j} \mathcal{F}_{\xi \rightarrow x}^{-1}\left(\chi_{\text {ext }}(\xi) \widehat{K_{1}}(t,|\xi|)|\xi|^{2 s+\omega}\right) *_{(x)} I_{\omega} u_{1}\right\|_{L^{q}} \\
\lesssim \mathrm{e}^{-c t} t^{-\kappa}\left\|I_{\omega} u_{1}\right\|_{W_{q}^{[2 s+\omega+2 \theta(j-\kappa-1)]^{+}} .}
\end{gathered}
$$

The proof follows after noticing that, applying Hardy-Littlewood-Sobolev theorem, we may deduce

$$
\left\|I_{\omega} u_{1}\right\|_{W_{q}^{[2 s+\omega+2 \theta(j-\kappa-1)]^{+}}} \lesssim\left\|u_{1}\right\|_{W_{m}^{[2 s+\omega+2 \theta(j-\kappa-1)]^{+}}} .
$$

Thus, the proof is complete.

To derive $L^{q}-L^{q}$ estimates for $q=1, \infty$, we cannot rely on Mikhlin-Hörmander multiplier theorem, so we prove that suitable multipliers associated with $M_{ \pm}$, are bounded in $L^{1}$ showing that their inverse Fourier transforms belong to $L^{1}$.

Proposition 3.4 Let $n \geqslant 1, s \geqslant 0$ a real number, $j \in \mathbb{N}$ and $k \in[0, j]$. Then, the solution to (8) localized at high frequencies verifies the estimates

$$
\left\|\partial_{t}^{j}(-\Delta)^{s} \chi_{\mathrm{ext}}(D) u(t, \cdot)\right\|_{L^{q}} \lesssim \mathrm{e}^{-c t} t^{-(j-k)}\left\|u_{1}\right\|_{W_{q}^{2[\theta k+s-\theta+1 / 2]^{+}},}
$$

for $q=1, \infty$ and any $t>0$, for some positive constant $c$.

Proof Using the decomposition (35), we would like to prove the following estimates:

$$
\begin{gathered}
\left\|\partial_{t}^{j}(-\Delta)^{s}\left(M_{+} u_{1}\right)(t, \cdot)\right\|_{L^{q}} \lesssim \mathrm{e}^{-c t} t^{-(j-k)}\left\|u_{1}\right\|_{W_{q}^{2[\theta k+s-\theta+1 / 2]^{+}}}, \\
\left\|\partial_{t}^{j}(-\Delta)^{s}\left(M_{-} u_{1}\right)(t, \cdot)\right\|_{L^{q}} \lesssim \mathrm{e}^{-c t} t^{-(j-k)}\left\|u_{1}\right\|_{W_{q}^{2[(1-\theta) k+s-\theta+1 / 2]^{+}}},
\end{gathered}
$$

for $q=1, \infty$ and $0 \leqslant k \leqslant j$.

First of all, we fix $j \geqslant k \geqslant 0$ and $s \geqslant 0$, and we consider the multiplier

$$
\hat{a}_{j, k, s}(t, \xi)=\frac{\mathrm{e}^{\lambda_{+}(|\xi|) t} \lambda_{+}(|\xi|)^{j}|\xi|^{2 s}}{\left(\lambda_{-}(|\xi|)-\lambda_{+}(|\xi|)\right)\left(1+|\xi|^{2}\right)^{\lceil s+\theta k-\theta+1 / 2\rceil^{+}}} \chi_{\text {ext }}(\xi),
$$

so that

$$
\partial_{t}^{j}(-\Delta)^{s}\left(M_{+} u_{1}\right)(t, x)=a_{j, k, s}(t, x) *_{(x)}(1-\Delta)^{\lceil s+\theta k-\theta+1 / 2\rceil^{+}} u_{1}(x),
$$

where $a_{j, k, s}=\mathcal{F}_{\xi \rightarrow x}^{-1}\left(\hat{a}_{j, k, s}(t, \xi)\right)$. To derive (45), we prove that

$$
\left\|a_{j, k, s}(t, \cdot)\right\|_{L^{1}} \lesssim \mathrm{e}^{-c t} t^{-(j-k)} \quad \text { for } \quad k=0,1 .
$$

We first consider $|x|<1 / N$. As in [19], we use the identity

$$
\sum_{j=1}^{n} \frac{x_{j}}{i|x|^{2}} \frac{\partial}{\partial \xi_{j}} \mathrm{e}^{i x \cdot \xi}=\mathrm{e}^{i x \cdot \xi}
$$

to integrate by parts $n-1$ times the function 


$$
a_{j, k, s}(t, \cdot)=\frac{1}{(2 \pi)^{n}} \int_{\mathbb{R}^{n}} e^{i x \cdot \xi} \hat{a}_{j, k, s}(t, \xi) d \xi
$$

Since the boundary terms vanish since $\chi_{\text {ext }}$ vanishes near $\{|\xi|=N\}$, we obtain

$$
\begin{aligned}
a_{j, k, s}(t, x) & =\frac{1}{(2 \pi)^{n}} \sum_{\ell=1}^{n} \frac{i x_{\ell}}{|x|^{2}} \int_{|\xi| \geqslant N} \mathrm{e}^{i x \cdot \xi} \partial_{\xi_{\ell}} \hat{a}_{j, k, s}(t, \xi) \mathrm{d} \xi \\
& =\frac{1}{(2 \pi)^{n}} \sum_{\ell=1}^{n} \sum_{i=1}^{n} \frac{-x_{\ell} x_{i}}{|x|^{4}} \int_{|\xi| \geqslant N} \mathrm{e}^{i x \cdot \xi} \partial_{\xi_{\ell}} \partial_{\xi_{i}} \hat{a}_{j, k, s}(t, \xi) \mathrm{d} \xi \\
& =\ldots \\
& =\frac{1}{(2 \pi)^{n}} \sum_{|\beta|=n-1}\left(\frac{i x}{|x|^{2}}\right)^{\beta} \int_{|\xi| \geqslant N} \mathrm{e}^{i x \cdot \xi} \partial_{\xi}^{\beta} \hat{a}_{j, k, s}(t, \xi) \mathrm{d} \xi .
\end{aligned}
$$

In particular, we can split each of the integrals of $a_{j, k, s}$ into two parts, which gives

$$
a_{j, k, s}(t, x)=\mathcal{I}_{1}(t, x)+\mathcal{I}_{2}(t, x),
$$

where

$$
\begin{aligned}
& \mathcal{I}_{1}(t, x)=\frac{1}{(2 \pi)^{n}} \sum_{|\beta|=n-1}\left(\frac{i x}{|x|^{2}}\right)^{\beta} \int_{N \leqslant|\xi| \leqslant 1 /|x|} \mathrm{e}^{i x \cdot \xi} \partial_{\xi}^{\beta} \hat{a}_{j, k, s}(t, \xi) \mathrm{d} \xi, \\
& \mathcal{I}_{2}(t, x)=\frac{1}{(2 \pi)^{n}} \sum_{|\beta|=n-1}\left(\frac{i x}{|x|^{2}}\right)^{\beta} \int_{|\xi| \geqslant 1 /|x|} \mathrm{e}^{i x \cdot \xi} \partial_{\xi}^{\beta} \hat{a}_{j, k, s}(t, \xi) \mathrm{d} \xi .
\end{aligned}
$$

Using (36) we find that for each exponent $h>0$, it holds

$$
|\xi|^{2 \theta h} \mathrm{e}^{\lambda_{+}(|\xi|) t} \lesssim t^{-h} \mathrm{e}^{-c t}
$$

which gives immediately

$$
\left|\partial_{\xi}^{\beta} \hat{a}_{j, k, s}(t, \xi)\right| \lesssim \mathrm{e}^{-c t} t^{-(j-k)}|\xi|^{-|\beta|-1},
$$

for any $k \leqslant j$. Hence, we obtain

$$
\begin{aligned}
\left|\mathcal{I}_{1}(t, x)\right| & \lesssim \mathrm{e}^{-c t} t^{-(j-k)}|x|^{-(n-1)} \int_{N \leqslant|\xi| \leqslant 1 /|x|}|\xi|^{-n} \mathrm{~d} \xi \\
& \lesssim \mathrm{e}^{-c t} t^{-(j-k)}|x|^{-(n-1)} \ln \left((N|x|)^{-1}\right) .
\end{aligned}
$$

Concerning $\mathcal{I}_{2}$, integrating by parts one more time, it yields

$$
\begin{aligned}
\mathcal{I}_{2}(t, x)= & \frac{1}{(2 \pi)^{n}} \sum_{|\beta|=n-1}\left(\frac{i x}{|x|^{2}}\right)^{\beta} \sum_{j=1}^{n} \frac{-i x_{j}}{|x|^{2}} \int_{|\xi|=1 /|x|} \mathrm{e}^{i x \cdot \xi} \partial_{\xi}^{\beta} \hat{a}_{j, k, s}(t, \xi) \mathrm{d} S_{\sigma} \\
& +\frac{1}{(2 \pi)^{n}} \sum_{|\beta|=n-1}\left(\frac{i x}{|x|^{2}}\right)^{\beta} \sum_{j=1}^{n} \frac{i x_{j}}{|x|^{2}} \int_{|\xi| \geqslant 1 /|x|} \mathrm{e}^{i x \cdot \xi} \partial_{\xi j} \partial_{\xi}^{\beta} \hat{a}_{j, k, s}(t, \xi) \mathrm{d} \xi .
\end{aligned}
$$

Following a similar procedure as for (49), we can derive 


$$
\begin{aligned}
\left|\mathcal{I}_{2}(t, x)\right| & \lesssim \mathrm{e}^{-c t} t^{-(j-k)}|x|^{-n}\left(\int_{|\xi|=1 /|x|}|\xi|^{-n} \mathrm{~d} S_{\sigma}+\int_{|\xi| \geqslant 1 /|x|}|\xi|^{-n-1} \mathrm{~d} \xi\right) \\
& \lesssim \mathrm{e}^{-c t} t^{-(j-k)}|x|^{-n+1}
\end{aligned}
$$

We now consider the remaining case $|x| \geqslant 1 / N$. Using the identity (48) and integrating by parts $n+1$ times, we may deduce

$$
\left|a_{j, k, s}(t, x)\right| \lesssim \mathrm{e}^{-c t} t^{-(j-k)}|x|^{-(n+1)} \int_{|\xi| \geqslant N}|\xi|^{-n-2} \mathrm{~d} \xi \lesssim \mathrm{e}^{-c t} t^{-(j-k)}|x|^{-(n+1)} .
$$

The combination of (50) and (51) concludes the proof of (47), and then (45) follows.

In order to prove (46), we proceed as before, setting

$$
\begin{aligned}
\mathcal{F}_{x \rightarrow \xi}\left(b_{j, k, s}(t, x)\right) & =\hat{b}_{j, k, s}(t, \xi) \\
& =\frac{\mathrm{e}^{\lambda_{-}(|\xi|) t} \lambda_{-}(|\xi|)^{j}|\xi|^{2 s}}{\left(\lambda_{+}(|\xi|)-\lambda_{-}(|\xi|)\right)\left(1+|\xi|^{2}\right)^{[s+(1-\theta) k-\theta+1 / 2\rceil^{+}}} \chi_{\text {ext }}(\xi) .
\end{aligned}
$$

Then, we have

$$
\partial_{t}^{j}(-\Delta)^{s}\left(M_{-} u_{1}\right)(t, x)=b_{j, k, s}(t, x) *_{(x)}(1-\Delta)^{\lceil s+(1-\theta) k-\theta+1 / 2\rceil^{+}} u_{1}(x) .
$$

Using (36) again we may observe that for each exponent $h>0$, it holds

$$
|\xi|^{2(1-\theta) h} \mathrm{e}^{\lambda_{-}(\xi) t} \lesssim t^{-h} \mathrm{e}^{-c t},
$$

so that

$$
\left|\partial_{\xi}^{\beta} \hat{b}_{j, k, s}(t, \xi)\right| \lesssim \mathrm{e}^{-c t} t^{-(j-k)}|\xi|^{-|\beta|-1},
$$

for any $k \leqslant j$. Repeating the procedure in the above, we arrive at

$$
\left\|b_{j, k, s}(t, \cdot)\right\|_{L^{1}} \lesssim \mathrm{e}^{-c t} t^{-(j-k)} .
$$

This concludes the proof of our desired estimate (46).

\subsection{Summary of $\left(L^{r} \cap L^{m}\right)-L^{q}$ estimates}

Thanks to Propositions 3.1-3.3 we may deduce the following estimates that will be used later to prove our global (in time) existence results.

- For any $q \in[1, \infty]$, we obtain the $L^{q}-L^{q}$ estimate

$$
\|u(t, \cdot)\|_{L^{q}} \lesssim(1+t)\left\|u_{1}\right\|_{L^{q}},
$$

as a consequence of Proposition 3.1 with $r=q$, and Proposition 3.2 if $q \neq 1, \infty$, or Proposition 3.4 if $q=1, \infty$, where $j=0$ and $s=0$.

- Let $q \in(1, \infty), m \in(1, q]$ and $r \in[1, m]$ such that $n(1 / r-1 / q) \leqslant 2 \rho$. Then, it holds that 


$$
\|u(t, \cdot)\|_{L^{q}} \lesssim(1+t)^{1-\frac{n}{2 \rho}\left(\frac{1}{r}-\frac{1}{q}\right)}\left\|u_{1}\right\|_{L^{r}}+\mathrm{e}^{-c t}\left\|u_{1}\right\|_{L^{m}}
$$

as a consequence of Propositions 3.1, and 3.3 with $\kappa=0$, where $j=0$ and $s=0$. In particular, we notice that

$$
n\left(\frac{1}{m}-\frac{1}{q}\right) \leqslant n\left(\frac{1}{r}-\frac{1}{q}\right) \leqslant 2 \rho<2 \theta .
$$

- For any $r \in[1,2]$, we obtain the estimate

$$
\left\|(-\Delta)^{s} u(t, \cdot)\right\|_{L^{2}} \lesssim(1+t)^{-\frac{n}{2(1-\rho)}\left(\frac{1}{r}-\frac{1}{2}\right)-\frac{s-\rho}{1-\rho}}\left\|u_{1}\right\|_{L^{r}}+\mathrm{e}^{-c t}\left\|u_{1}\right\|_{L^{2}},
$$

as a consequence of Propositions 3.1, and 3.2 with $\kappa=0$, where $q=2, j=0$ and $s \in[\rho, \theta]$. In particular, for $s=\theta$, we get

$$
\left\|(-\Delta)^{\theta} u(t, \cdot)\right\|_{L^{2}} \lesssim(1+t)^{-\frac{n}{2(1-\rho)}\left(\frac{1}{r}-\frac{1}{2}\right)-\frac{\theta-\rho}{1-\rho}}\left\|u_{1}\right\|_{L^{r}}+\mathrm{e}^{-c t}\left\|u_{1}\right\|_{L^{2}} .
$$

- For any $r \in[1,2]$, we may estimate

$$
\left\|u_{t}(t, \cdot)\right\|_{L^{2}} \lesssim(1+t)^{\max \left\{-\frac{n}{2(1-\rho)}\left(\frac{1}{r}-\frac{1}{2}\right)-1+\frac{\rho}{1-\rho},-\frac{n}{2 \rho}\left(\frac{1}{r}-\frac{1}{2}\right)\right\}}\left\|u_{1}\right\|_{L^{r}}+\mathrm{e}^{-c t}\left\|u_{1}\right\|_{L^{2}},
$$

as a consequence of Propositions 3.1, and 3.2 with $\kappa=0$, where $j=1, s=0$ and $q=2$.

- Let $q \in(1, \infty), m \in(1, q]$ and $r \in[1, m]$, be such that $n(1 / r-1 / q) \leqslant 2 \rho$. Then

$$
\begin{aligned}
& \left\|u_{t}(t, \cdot)\right\|_{L^{q}} \\
& \quad \lesssim(1+t)^{-\frac{n}{2 \rho}\left(\frac{1}{r}-\frac{1}{q}\right)}\left\|u_{1}\right\|_{L^{r}}+\mathrm{e}^{-c t} t^{-\frac{n}{2 \theta}\left(\frac{1}{m}-\frac{1}{q}\right)}\left\|u_{1}\right\|_{L^{m}},
\end{aligned}
$$

follows by Propositions 3.1 and 3.3 , setting $2 \theta \kappa=n(1 / m-1 / q)$, where $j=1$ and $s=0$. Estimate (58) will be used to prove Theorem 2.3, and we stress that this estimate is singular at $t=0$ if $m<q$.

\section{Proof of the global (in time) existence results}

\subsection{Philosophy of our approach}

Let us first introduce some notation for the proof of the global (in time) existence of small data solutions. Throughout this section, $K_{1}(t, x)$ denotes the fundamental solution to the linear Cauchy problem (29) with initial data $u_{0}=0$ and $u_{1}=\delta_{0}$, where $\delta_{0}$ is the Dirac distribution in $x=0$ with respect to the spatial variables. As a consequence, we may represent the solution to the Cauchy problem (8) in the form

$$
u^{\operatorname{lin}}(t, x)=K_{1}(t, x) *_{(x)} u_{1}(x) .
$$

We may introduce the operator

$$
N: u \in X(T) \rightarrow N u(t, x)=u^{\operatorname{lin}}(t, x)+u^{\text {non }}(t, x),
$$


where $X(T)$ is an evolution space which will be defined in a suitable way in the proof of each theorem, and $u^{\text {non }}=u^{\text {non }}(t, x)$ is an integral operator with the following representation

$$
u^{\mathrm{non}}(t, x)=\int_{0}^{t} K_{1}(t-s, x) *_{(x)} F\left(u, u_{t}\right)(s, x) \mathrm{d} s .
$$

According to the Duhamel's principle, we will prove the existence of the unique global (in time) solution to (1) as the fixed point of the operator $N$. Hence, in order to get the global (in time) existence and uniqueness of the solution in $X(T)$, we need to prove the following two crucial estimates:

$$
\begin{gathered}
\|N u\|_{X(T)} \leqslant C\left\|u_{1}\right\|_{\mathcal{A}}+\|u\|_{X(T)}^{p}, \\
\|N u-N v\|_{X(T)} \leqslant C\|u-v\|_{X(T)}\left(\|u\|_{X(T)}^{p-1}+\|v\|_{X(T)}^{p-1}\right),
\end{gathered}
$$

with $C>0$, independent of $T$, where $\mathcal{A}$ denotes the space of the datum. As a consequence of Banach's fixed point theorem, the conditions (60) and (61) guarantee the existence of a uniquely determined solution $u$ to (1) that is $u$ solves the integral equation $u=u^{\text {lin }}+u^{\text {non }}$. We simultaneously gain a local and a global (in time) existence result.

Indeed, let $R>0$ be such that $C R^{p-1}<1 / 2$. Then $N$ is a contraction on $X_{R}(T)=\left\{u \in X(T):\|u\|_{X(T)} \leqslant R\right\}$, thanks to (61). The solution to (1) is a fixed point for $N$, so if $\left\|u^{\operatorname{lin}}\right\|_{X(T)} \leqslant R / 2$, then $u \in X_{R}(T)$, thanks to (60). As a consequence, the uniqueness and existence of the solution in $X_{R}(T)$ follows by the Banach fixed point theorem on contractions. The condition $\left\|u^{\operatorname{lin}}\right\|_{X(T)} \leqslant R / 2$ is obtained taking initial data verifying $\left\|u_{1}\right\|_{\mathcal{A}} \leqslant \epsilon$, with $\epsilon$ such that $C \epsilon \leqslant R / 2$. Since $C, R$ and $\epsilon$ do not depend on $T$, the solution is global (in time).

In the proof of our global (in time) existence results, the following proposition will be useful, which use goes back to [29].

Proposition 4.1 Let $\alpha, \beta \in \mathbb{R}$. Then,

$$
\int_{0}^{t}(1+t-s)^{-\alpha}(1+s)^{-\beta} \mathrm{d} s \lesssim \begin{cases}(1+t)^{-\min \{\alpha, \beta\}} & \text { if } \max \{\alpha, \beta\}>1 \\ (1+t)^{-\min \{\alpha, \beta\}} \log (2+t) & \text { if } \max \{\alpha, \beta\}=1 \\ (1+t)^{1-\alpha-\beta} & \text { if } \max \{\alpha, \beta\}<1\end{cases}
$$

\subsection{Proof of Theorem 2.1}

Let us define the evolution space

$$
X(T)=\mathcal{C}\left([0, T], L^{1} \cap H^{2 \theta}\right) \cap \mathcal{C}^{1}\left([0, T], L^{2}\right)
$$

with its corresponding norm

$$
\begin{aligned}
\|u\|_{X(T)}=\sup _{t \in[0, T]}\left((1+t)^{-1}\|u(t, \cdot)\|_{L^{1}}+\|u(t, \cdot)\|_{L^{\bar{q}}}\right. & \\
& \left.+(1+t)^{\frac{n}{4(1-\rho)}}+\frac{\theta-\rho}{1-\rho}\left\|(-\Delta)^{\theta} u(t, \cdot)\right\|_{L^{2}}+(1+t)^{\gamma_{2}+1}\left\|u_{t}(t, \cdot)\right\|_{L^{2}}\right),
\end{aligned}
$$


where we define

$$
\begin{aligned}
\gamma_{q} & =\min \left\{\frac{n}{2(1-\rho)}\left(1-\frac{1}{q}\right)-\frac{\rho}{1-\rho}, \frac{n}{2 \rho}\left(1-\frac{1}{q}\right)-1\right\} \\
& = \begin{cases}\frac{n}{2(1-\rho)}\left(1-\frac{1}{q}\right)-\frac{\rho}{1-\rho} & \text { if } q \geqslant \bar{q}, \\
\frac{n}{2 \rho}\left(1-\frac{1}{q}\right)-1 & \text { if } q \leqslant \bar{q} .\end{cases}
\end{aligned}
$$

We also recall that $\bar{q}=n /(n-2 \rho)$.

Defining the data space $\mathcal{A}=L^{1} \cap L^{2}$, it follows immediately

$$
\left\|u^{\operatorname{lin}}\right\|_{X(T)} \lesssim\left\|u_{1}\right\|_{\mathcal{A}},
$$

and so we conclude that $u^{\text {lin }} \in X(T)$. Indeed, it is sufficient to apply (53) with $q=1,(54)$ with $q=\bar{q}, r=1$ and $m \in(1, \min \{\bar{q}, 2\}]$, (56) with $r=1$, and (57) with $r=1$.

In the remaining part of the proof we will estimate $u^{\text {non }}$ in the norm of $X(T)$. To do this, let us introduce the following useful lemma.

Lemma 4.1 Let $u \in X(t)$. Then, for any $q \in[\bar{q}, 2 p]$, we have

$$
\|u(t, \cdot)\|_{L^{q}} \lesssim(1+t)^{-\frac{n}{2(1-\rho)}\left(1-\frac{1}{q}\right)+\frac{\rho}{1-\rho}}\|u\|_{X(t)}
$$

for any $t \geqslant 0$.

Proof In the limit case $q=\bar{q}$, the estimate (64) is an obvious consequence of $\|u\|_{X(t)}<\infty$. On the other hand, due to the restriction $p \leqslant n /(n-4 \theta)$ if $4 \theta<n$, the Sobolev embed$\operatorname{ding} H^{2 \theta} \hookrightarrow L^{2 p}$ holds, and (64) follows as a consequence of Gagliardo-Nirenberg inequality.

Thanks to (53) with $q=1$, applying the Minkowski's integral inequality, we get

$$
\left\|u^{\mathrm{non}}(t, \cdot)\right\|_{L^{1}} \lesssim \int_{0}^{t}(1+t-s)\left\||u(s, \cdot)|^{p}\right\|_{L^{1}} \mathrm{~d} s .
$$

Due to $p>p_{0}(n, \rho)=1+2 /(n-2 \rho)>\bar{q}$, we can apply (64) with $q=p$ to get

$$
\left\||u(s, \cdot)|^{p}\right\|_{L^{1}}=\|u(s, \cdot)\|_{L^{p}}^{p} \lesssim(1+s)^{-\frac{n(p-1)}{2(1-\rho)}+\frac{p \rho}{1-\rho}}\|u\|_{X(s)}^{p} .
$$

In particular, using again $p>p_{0}(n, \rho)$, we obtain

$$
\frac{n(p-1)}{2(1-\rho)}-\frac{p \rho}{1-\rho}=\frac{(n-2 \rho)(p-1)-2 \rho}{2(1-\rho)}>\frac{(n-2 \rho)\left(p_{0}(n, \rho)-1\right)-2 \rho}{2(1-\rho)}=1 .
$$

Therefore, applying Proposition 4.1 we conclude for $t \leqslant T$ that

$$
\left\|u^{\mathrm{non}}(t, \cdot)\right\|_{L^{1}} \lesssim(1+t)\|u\|_{X(T)}^{p} .
$$

In order to estimate $u^{\text {non }}(t, \cdot)$ in the $L^{\bar{q}}$ space, we apply (54) with $q=\bar{q}, r=1$ and $m \in(1, \min \{\bar{q}, 2\}]$. Noticing that $m p \leqslant 2 p$, we can apply (64) with $q=p$ and with $q=m p$, to get 


$$
\begin{aligned}
\left\|u^{\text {non }}(t, \cdot)\right\|_{L^{\bar{q}}} & \lesssim \int_{0}^{t}\left\||u(s, \cdot)|^{p}\right\|_{L^{1} \cap L^{m}} \mathrm{~d} s=\int_{0}^{t}\left(\|u(s, \cdot)\|_{L^{p}}^{p}+\|u(s, \cdot)\|_{L^{m p}}^{p}\right) \mathrm{d} s \\
& \lesssim\|u\|_{X(T)}^{p} \int_{0}^{t}(1+s)^{-\frac{n(p-1)}{2(1-\rho)}+\frac{p \rho}{1-\rho}} \mathrm{d} s \lesssim\|u\|_{X(T)}^{p},
\end{aligned}
$$

where in the last inequality we used again (65) and Proposition 4.1.

In order to estimate $\left\|(-\Delta)^{\theta} u^{\text {non }}(t, \cdot)\right\|_{L^{2}}$ we now apply (56) with $r=1$ in $[0, t / 2]$ and with $r=2$ in $[t / 2, t]$. Using (64) with $q=p$ and with $q=2 p$, noticing that

$$
\left\||u(s, \cdot)|^{p}\right\|_{L^{2}}=\|u(s, \cdot)\|_{L^{2 p}}^{p} \lesssim(1+s)^{-\frac{n(p-1)}{2(1-\rho)}+\frac{p \rho}{1-\rho}-\frac{n}{4(1-\rho)}}\|u\|_{X(s)}^{p},
$$

we derive

$$
\begin{aligned}
\left\|(-\Delta)^{\theta} u^{\mathrm{non}}(t, \cdot)\right\|_{L^{2}} \lesssim & \int_{0}^{t / 2}(1+t-s)^{-\frac{n}{4(1-\rho)}-\frac{\theta-\rho}{1-\rho}}\left\||u(s, \cdot)|^{p}\right\|_{L^{1} \cap L^{2}} \mathrm{~d} s \\
& +\int_{t / 2}^{t}(1+t-s)^{-\frac{\theta-\rho}{1-\rho}}\left\||u(s, \cdot)|^{p}\right\|_{L^{2}} \mathrm{~d} s \\
\lesssim & (1+t)^{-\frac{n}{4(1-\rho)}-\frac{\theta-\rho}{1-\rho}} \int_{0}^{t / 2}(1+s)^{-\frac{n(p-1)}{2(1-\rho)}+\frac{p \rho}{1-\rho}} \mathrm{d} s\|u\|_{X(T)}^{p} \\
& +(1+t)^{-\frac{n(p-1)}{2(1-\rho)}+\frac{p \rho}{1-\rho}-\frac{n}{4(1-\rho)}} \int_{t / 2}^{t}(1+t-s)^{-\frac{\theta-\rho}{1-\rho}} \mathrm{d} s\|u\|_{X(T)}^{p} .
\end{aligned}
$$

Here, we used $(1+t-s) \approx(1+t)$ for any $s \in[0, t / 2]$ and $(1+s) \approx(1+t)$ for any $s \in[t / 2, t]$. Again, we plan to use (65). The first consequence is that

$$
\int_{0}^{t / 2}(1+s)^{-\frac{n(p-1)}{2(1-\rho)}+\frac{p \rho}{1-\rho}} \mathrm{d} s \leqslant \int_{0}^{\infty}(1+s)^{-\frac{n(p-1)}{2(1-\rho)}+\frac{p \rho}{1-\rho}} \mathrm{d} s=C .
$$

On the other hand, due to the fact that $(\theta-\rho) /(1-\rho) \leqslant 1$, using (65) we get

$$
(1+t)^{-\frac{n(p-1)}{2(1-\rho)}+\frac{p \rho}{1-\rho}-\frac{n}{4(1-\rho)}} \int_{t / 2}^{t}(1+t-s)^{-\frac{\theta-\rho}{1-\rho}} \mathrm{d} s \lesssim(1+t)^{-\frac{n}{4(1-\rho)}-\frac{\theta-\rho}{1-\rho}} .
$$

Summarizing the previous estimates, we proved the desired estimate

$$
\left\|(-\Delta)^{\theta} u^{\text {non }}(t, \cdot)\right\|_{L^{2}} \lesssim(1+t)^{-\frac{n}{4(1-\rho)}-\frac{\theta-\rho}{1-\rho}}\|u\|_{X(T)}^{p} .
$$

To estimate the time derivative of the solution, we distinguish two cases. If $2<\bar{q}$, that is, $n<4 \rho$ and

$$
\gamma_{2}=\frac{n}{4 \rho}-1
$$

we may apply (57) with $r=1$ to estimate 


$$
\begin{aligned}
\left\|u_{t}^{\text {non }}(t, \cdot)\right\|_{L^{2}} & \lesssim \int_{0}^{t}(1+t-s)^{-\frac{n}{4 \rho}}\left\||u(s, \cdot)|^{p}\right\|_{L^{1} \cap L^{2}} \mathrm{~d} s \\
& \lesssim \int_{0}^{t}(1+t-s)^{-\frac{n}{4 \rho}}(1+s)^{-\frac{n(p-1)}{2(1-\rho)}+\frac{p \rho}{1-\rho}} \mathrm{d} s\|u\|_{X(T)}^{p} \\
& \lesssim(1+t)^{-\frac{n}{4 \rho}}\|u\|_{X(T)}^{p},
\end{aligned}
$$

using (64) with $q=p$ and with $q=2 p$, (65) and Proposition 4.1.

Now let $2 \geqslant \bar{q}$, that is, $n \geqslant 4 \rho$ and

$$
\gamma_{2}=\frac{n}{4(1-\rho)}-\frac{\rho}{1-\rho} .
$$

We fix $r^{*} \in(1,2)$ such that

$$
\frac{n}{2}\left(\frac{1}{r^{*}}-\frac{1}{2}\right)=\rho
$$

and we apply (57) with $r=1$ in $[0, t / 2]$ and with $r=r^{*}$ in $[t / 2, t]$. Then, we obtain

$$
\begin{aligned}
\left\|u_{t}^{\text {non }}(t, \cdot)\right\|_{L^{2}} \lesssim & \int_{0}^{t / 2}(1+t-s)^{-\frac{n}{4(1-\rho)}+\frac{\rho}{1-\rho}-1}\left\||u(s, \cdot)|^{p}\right\|_{L^{1} \cap L^{2}} \mathrm{~d} s \\
& +\int_{t / 2}^{t}(1+t-s)^{-1}\left\||u(s, \cdot)|^{p}\right\|_{L^{r^{*}} \cap L^{2}} \mathrm{~d} s \\
\lesssim & (1+t)^{-\frac{n}{4(1-\rho)}+\frac{\rho}{1-\rho}-1} \int_{0}^{t / 2}(1+s)^{-\frac{n(p-1)}{2(1-\rho)}+\frac{p \rho}{1-\rho}} \mathrm{d} s\|u\|_{X(T)}^{p} \\
& +(1+t)^{-\frac{n(p-1)}{2(1-\rho)}+\frac{p \rho}{1-\rho}-\frac{n}{2(1-\rho)}\left(1-\frac{1}{r^{*}}\right)} \log (2+t)\|u\|_{X(T)}^{p},
\end{aligned}
$$

where we applied (64) carrying $q=r^{*} p$ to obtain

$$
\left\||u(s, \cdot)|^{p}\right\|_{L^{r^{*}} \cap L^{2}} \lesssim(1+s)^{-\frac{n(p-1)}{2(1-\rho)}+\frac{p \rho}{1-\rho}-\frac{n}{2(1-\rho)}\left(1-\frac{1}{r^{*}}\right)}\|u\|_{X(T)}^{p} .
$$

Again, we use (65) in two ways: to get (66), and to estimate

$$
\begin{aligned}
-\frac{n(p-1)}{2(1-\rho)}+\frac{p \rho}{1-\rho}-\frac{n}{2(1-\rho)}\left(1-\frac{1}{r^{*}}\right) & <-1-\frac{n}{2(1-\rho)}\left(1-\frac{1}{r^{*}}\right) \\
& =-\frac{n}{4(1-\rho)}+\frac{\rho}{1-\rho}-1,
\end{aligned}
$$

so that we obtain the desired estimate

$$
\left\|u_{t}^{\text {non }}(t, \cdot)\right\|_{L^{2}} \lesssim(1+t)^{-\frac{n}{4(1-\rho)}+\frac{\rho}{1-\rho}-1}\|u\|_{X(T)}^{p} .
$$

Summarizing, we proved

$$
\left\|u^{\mathrm{non}}\right\|_{X(T)} \lesssim\|u\|_{X(T)}^{p},
$$

and this concludes the proof of (60).

In a similar way, we can prove (61). Indeed, 


$$
\begin{aligned}
& \left\|\partial_{t}^{j}(-\Delta)^{\theta k}(N u-N v)(t, \cdot)\right\|_{L^{q}} \\
& \quad=\left\|\partial_{t}^{j}(-\Delta)^{\theta k} \int_{0}^{t} K_{1}(t-s, \cdot) *_{(x)}\left(|u(s, \cdot)|^{p}-|v(s, \cdot)|^{p}\right) \mathrm{d} s\right\|_{L^{q}}
\end{aligned}
$$

for $j=0,1$ and $k=0,1$. We proceed as we did to prove (67), but we now use Hölder's inequality to estimate

$$
\left\||u(s, \cdot)|^{p}-|v(s, \cdot)|^{p}\right\|_{L^{r}} \lesssim\|u(s, \cdot)-v(s, \cdot)\|_{L^{r p}}\left(\|u(s, \cdot)\|_{L^{r p}}^{p-1}+\|v(s, \cdot)\|_{L^{r p}}^{p-1}\right),
$$

so that it is sufficient to replace $\|u\|_{X(T)}^{p}$ by $\|u-v\|_{X(T)}\left(\|u\|_{X(T)}^{p-1}+\|v\|_{X(T)}^{p-1}\right)$, in each step of the proof.

This completes the proof of Theorem 2.1.

\subsection{Proof of Theorem 2.2}

We define the solution space

$$
X(T)=\mathcal{C}\left([0, T], L^{\eta} \cap H^{2 \theta}\right) \cap \mathcal{C}^{1}\left([0, T], L^{2}\right) .
$$

For $p>p_{0}(n / \eta, \rho)$, we equip $X(T)$ with the norm

$$
\begin{aligned}
\|u\|_{X(T)}= & \sup _{t \in[0, T]}\left((1+t)^{-1}\|u(t, \cdot)\|_{L^{\eta}}+\|u(t, \cdot)\|_{L^{\bar{q}}}\right. \\
& +(1+t)^{\frac{n}{2(1-\rho)}\left(\frac{1}{\eta}-\frac{1}{2}\right)+\frac{\theta-\rho}{1-\rho}}\left\|(-\Delta)^{\theta} u(t, \cdot)\right\|_{L^{2}} \\
& \left.+(1+t)^{\gamma_{\eta, 2}}\left\|u_{t}(t, \cdot)\right\|_{L^{2}}\right),
\end{aligned}
$$

where we define

$$
\begin{aligned}
\gamma_{\eta, q} & =\min \left\{\frac{n}{2(1-\rho)}\left(\frac{1}{\eta}-\frac{1}{q}\right)-\frac{\rho}{1-\rho}, \frac{n}{2 \rho}\left(\frac{1}{\eta}-\frac{1}{q}\right)-1\right\} \\
& = \begin{cases}\frac{n}{2(1-\rho)}\left(\frac{1}{\eta}-\frac{1}{q}\right)-\frac{\rho}{1-\rho} & \text { if } q \geqslant \bar{q}, \\
\frac{n}{2 \rho}\left(\frac{1}{\eta}-\frac{1}{q}\right)-1 & \text { if } q \leqslant \bar{q} .\end{cases}
\end{aligned}
$$

Again, we recall that $\bar{q}=n \eta /(n-2 \rho \eta)$.

Let us take into consideration the case $p=p_{0}(n / \eta, \rho)$. In the case $\theta<1$ and $2<\bar{q}$, we use the same norm as before. In the case $\theta<1$ and $\bar{q} \geqslant 2$, we modify the norm in the above, replacing $(1+t)^{\gamma_{\eta, 2}}\left\|u_{t}(t, \cdot)\right\|_{L^{2}}$ with $(1+t)^{\gamma_{\eta, 2}}(\log (2+t))^{-1}\left\|u_{t}(t, \cdot)\right\|_{L^{2} \cdot \text { Namely, }}$

$$
\begin{aligned}
\|u\|_{X(T)}= & \sup _{t \in[0, T]}\left((1+t)^{-1}\|u(t, \cdot)\|_{L^{\eta}}+\|u(t, \cdot)\|_{L^{\bar{q}}}\right. \\
& +(1+t)^{\frac{n}{2(1-\rho)}\left(\frac{1}{\eta}-\frac{1}{2}\right)+\frac{\theta-\rho}{1-\rho}}\left\|(-\Delta)^{\theta} u(t, \cdot)\right\|_{L^{2}} \\
& \left.+(1+t)^{\frac{n}{2(1-\rho)}\left(\frac{1}{\eta}-\frac{1}{2}\right)-\frac{\rho}{1-\rho}}(\log (2+t))^{-1}\left\|u_{t}(t, \cdot)\right\|_{L^{2}}\right) .
\end{aligned}
$$


Considering $p=p_{0}(n / \eta, \rho)$ and $\theta=1$, we fix $s \in[\rho, 1)$ such that

$$
s \geqslant \frac{n}{4}\left(1-\frac{1}{p}\right)
$$

so that $H^{2 s} \hookrightarrow L^{2 p}$ and we modify the norm of $X(T)$ in the following way:

$$
\begin{aligned}
\|u\|_{X(T)}= & \sup _{t \in[0, T]}\left((1+t)^{-1}\|u(t, \cdot)\|_{L^{\eta}}+\|u(t, \cdot)\|_{L^{\bar{q}}}\right. \\
& +(1+t)^{\frac{n}{2(1-\rho)}\left(\frac{1}{\eta}-\frac{1}{2}\right)+\frac{s-\rho}{1-\rho}}\left\|(-\Delta)^{s} u(t, \cdot)\right\|_{L^{2}} \\
& +(1+t)^{\frac{n}{2(1-\rho)}\left(\frac{1}{\eta}-\frac{1}{2}\right)+1}(\log (2+t))^{-1}\|\Delta u(t, \cdot)\|_{L^{2}} \\
& \left.+(1+t)^{\gamma_{\eta, 2}}(\log (2+t))^{-i}\left\|u_{t}(t, \cdot)\right\|_{L^{2}}\right),
\end{aligned}
$$

where $i=0$ if $2<\bar{q}$ and $i=1$ if $\bar{q} \geqslant 2$.

As in the proof of Theorem 2.1, defining the data space $\mathcal{A}=L^{\eta} \cap L^{2}$, estimate (63) immediately follows, and so we conclude that $u^{\text {lin }} \in X(T)$. Indeed, it is sufficient to apply (53) with $q=\eta$, (54) with $q=\bar{q}, r=m=\eta$, (56) with $r=\eta$, and (57) with $r=\eta$. If $p=p_{0}(n / \eta, \rho)$ and $\theta=1$, we also use (55).

It remains to estimate $u^{\text {non }}$ in the $X(T)$ norm. Similarly to Lemma 4.1, the next lemma can be obtained.

Lemma 4.2 Let $u \in X(t)$. Then, for any $q \in[\bar{q}, 2 p]$, we have:

$$
\|u(t, \cdot)\|_{L^{q}} \lesssim(1+t)^{-\frac{n}{2(1-\rho)}\left(\frac{1}{\eta}-\frac{1}{q}\right)+\frac{\rho}{1-\rho}}\|u\|_{X(t)}
$$

for any $t \geqslant 0$.

First, let $p>p_{0}(n / \eta, \rho)$. In this case, it is sufficient to follow the proof of Theorem 2.1, replacing the $L^{1}$ smallness of the initial datum by the $L^{\eta}$ smallness.

For instance, thanks to (53) with $q=\eta$, applying the Minkowski's integral inequality, one gets

$$
\left\|u^{\mathrm{non}}(t, \cdot)\right\|_{L^{\eta}} \lesssim \int_{0}^{t}(1+t-s)\left\||u(s, \cdot)|^{p}\right\|_{L^{\eta}} \mathrm{d} s .
$$

Since the fact that $\eta p>\eta p_{0}(n / \eta, \rho)>\bar{q}$, we can apply (69) with $q=\eta p$ to get

$$
\left\||u(s, \cdot)|^{p}\right\|_{L^{\eta}}=\|u(s, \cdot)\|_{L^{\eta p}}^{p} \lesssim(1+s)^{-\frac{n(p-1)}{2 \eta(1-\rho)}+\frac{p \rho}{1-\rho}}\|u\|_{X(s)}^{p} .
$$

In particular, using again $p>p_{0}(n / \eta, \rho)$, we obtain (65) with $n / \eta$ in place of $n$, namely

$$
\begin{aligned}
\frac{n(p-1)}{2 \eta(1-\rho)}-\frac{p \rho}{1-\rho} & =\frac{(n / \eta-2 \rho)(p-1)-2 \rho}{2(1-\rho)} \\
& >\frac{(n / \eta-2 \rho)\left(p_{0}(n / \eta, \rho)-1\right)-2 \rho}{2(1-\rho)}=1 .
\end{aligned}
$$

Hence, using Proposition 4.1 we conclude 


$$
\left\|u^{\mathrm{non}}(t, \cdot)\right\|_{L^{\eta}} \lesssim(1+t)\|u\|_{X(T)}^{p} .
$$

We omit the steps of the proof used to estimate $u^{\text {non }}(t, \cdot)$ in $L^{q}$, and to estimate $u^{\text {non }}(t, \cdot)$ and $u_{t}^{\text {non }}(t, \cdot)$ in $L^{2}$, since they are analogous to those in the proof of Theorem 2.1.

Now we consider the critical case $p=p_{0}(n / \eta, \rho)$ in the following part. In this case, the strict inequality in (70) no longer holds, so we cannot follow the same proof of the supercritical case. For this reason, to deal with the critical case, we do not rely on estimate of $|u(t, \cdot)|^{p}$ in $L^{\eta}$, but in some $L^{\eta_{1}}$ spaces with $\eta_{1} \in[1, \eta)$. The same strategy is used in the context of semilinear damped waves in [30] and in the context of semilinear fractional diffusive equations in [31].

We fix $\eta_{1} \in(1, \eta)$, sufficiently close to $\eta$ to guarantee $n\left(1 / \eta_{1}-1 / \eta\right)<2 \rho$ and $\eta_{1} p_{0}(n / \eta, \rho) \geqslant \bar{q}$. As a consequence of Lemma 4.2 , it yields

$$
\begin{aligned}
\left\||u(s, \cdot)|^{p}\right\|_{L^{\eta_{1}}} & \lesssim(1+s)^{-\frac{n}{2(1-\rho)}\left(\frac{p}{\eta}-\frac{1}{\eta_{1}}\right)+\frac{p \rho}{1-\rho}}\|u\|_{X(T)} \\
& =(1+s)^{-1+\frac{n}{2(1-\rho)}\left(\frac{1}{\eta_{1}}-\frac{1}{\eta}\right)}\|u\|_{X(T)},
\end{aligned}
$$

where in the last equality we used $p=p_{0}(n / \eta, \rho)$. Using (54) with $q=\eta$ and $r=m=\eta_{1}$, we find

$$
\begin{aligned}
\left\|u^{\mathrm{non}}(t, \cdot)\right\|_{L^{\eta}} & \lesssim \int_{0}^{t}(1+t-s)^{1-\frac{n}{2 \rho}\left(\frac{1}{\eta_{1}}-\frac{1}{\eta}\right)}\left\||u(s, \cdot)|^{p}\right\|_{L^{\eta_{1}}} \mathrm{~d} s \\
& \lesssim \int_{0}^{t}(1+t-s)^{1-\frac{n}{2 \rho}\left(\frac{1}{\eta_{1}}-\frac{1}{\eta}\right)}(1+s)^{-1+\frac{n}{2(1-\rho)}\left(\frac{1}{\eta_{1}}-\frac{1}{\eta}\right)} \mathrm{d} s\|u\|_{X(T)}^{p} \\
& \lesssim(1+t)^{1-\frac{n}{2 \rho}\left(\frac{1}{\eta_{1}}-\frac{1}{\eta}\right)+\frac{n}{2(1-\rho)}\left(\frac{1}{\eta_{1}}-\frac{1}{\eta}\right)}\|u\|_{X(T)}^{p} \lesssim(1+t)\|u\|_{X(T)}^{p},
\end{aligned}
$$

where we used Proposition 4.1 with $\alpha, \beta<1$.

Since $\eta_{1}<\eta$, we find immediately

$$
1-\frac{n}{2 \rho}\left(\frac{1}{\eta_{1}}-\frac{1}{\bar{q}}\right)<-\frac{n}{2(1-\rho)}\left(\frac{1}{\eta_{1}}-\frac{1}{\bar{q}}\right)+\frac{\rho}{1-\rho} .
$$

Thus, using (54) with $q=\bar{q}$ and $r=m=\eta_{1}$, we obtain

$$
\begin{aligned}
\left\|u^{\mathrm{non}}(t, \cdot)\right\|_{L^{\bar{q}}} & \lesssim \int_{0}^{t}(1+t-s)^{-\frac{n}{2(1-\rho)}\left(\frac{1}{\eta_{1}}-\frac{1}{\bar{q}}\right)+\frac{\rho}{1-\rho}}\left\||u(s, \cdot)|^{p}\right\|_{L^{\eta_{1}}} \mathrm{~d} s \\
& \lesssim \int_{0}^{t}(1+t-s)^{-\frac{n}{2(1-\rho)}\left(\frac{1}{\eta_{1}}-\frac{1}{\eta}\right)}(1+s)^{-1+\frac{n}{2(1-\rho)}\left(\frac{1}{\eta_{1}}-\frac{1}{\eta}\right)} \mathrm{d} s\|u\|_{X(T)}^{p} \\
& \lesssim\|u\|_{X(T)}^{p} .
\end{aligned}
$$

In order to estimate $(-\Delta)^{\theta} u^{\text {non }}(t, \cdot)$ in the $L^{2}$ norm, we directly apply (56) with $r=\eta_{1}$ in $[0, t / 2]$ and with $r=2$ in $[t / 2, t]$. Using (69) with $q=\eta_{1} p$ and with $q=2 p$, noticing that

$$
\left\||u(s, \cdot)|^{p}\right\|_{L^{2}} \lesssim(1+s)^{-\frac{n}{2(1-\rho)}\left(\frac{p}{\eta}-\frac{1}{2}\right)+\frac{p \rho}{1-\rho}}\|u\|_{X(T)}=(1+s)^{-1-\frac{n}{2(1-\rho)}\left(\frac{1}{\eta}-\frac{1}{2}\right)}\|u\|_{X(T)},
$$

we obtain the desired estimate 


$$
\begin{aligned}
& \left\|(-\Delta)^{\theta} u^{\text {non }}(t, \cdot)\right\|_{L^{2}} \\
& \lesssim \int_{0}^{t / 2}(1+t-s)^{-\frac{n}{2(1-\rho)}\left(\frac{1}{\eta_{1}}-\frac{1}{2}\right)-\frac{\theta-\rho}{1-\rho}}\left\||u(s, \cdot)|^{p}\right\|_{L^{\eta_{1}} \cap L^{2}} \mathrm{~d} s \\
& +\int_{t / 2}^{t}(1+t-s)^{-\frac{\theta-\rho}{1-\rho}}\left\||u(s, \cdot)|^{p}\right\|_{L^{2}} \mathrm{~d} s \\
& \lesssim(1+t)^{-\frac{n}{2(1-\rho)}\left(\frac{1}{\eta_{1}}-\frac{1}{2}\right)-\frac{\theta-\rho}{1-\rho}} \int_{0}^{t / 2}(1+s)^{-1+\frac{n}{2(1-\rho)}\left(\frac{1}{\eta_{1}}-\frac{1}{\eta}\right)} \mathrm{d} s\|u\|_{X(T)}^{p} \\
& +(1+t)^{-1-\frac{n}{2(1-\rho)}\left(\frac{1}{\eta}-\frac{1}{2}\right)} \int_{t / 2}^{t}(1+t-s)^{-\frac{\theta-\rho}{1-\rho}} \mathrm{d} s\|u\|_{X(T)}^{p} \\
& \lesssim\left\{\begin{array}{l}
(1+t)^{-\frac{n}{2(1-\rho)}\left(\frac{1}{\eta}-\frac{1}{2}\right)-\frac{\theta-\rho}{1-\rho}}\|u\|_{X(T)}^{p} \\
(1+t)^{-\frac{n}{2(1-\rho)}\left(\frac{1}{\eta}-\frac{1}{2}\right)-1} \log (2+t)\|u\|_{X(T)}^{p} \text { if } \theta=1 .
\end{array}\right.
\end{aligned}
$$

In the case $\theta=1$, we proceed in a similar way to estimate $(-\Delta)^{s} u^{\text {non }}(t, \cdot)$ in the $L^{2}$ norm, but we use (55) rather than (56), and the logarithmic term does not appear, due to the fact that $s-\rho<1-\rho$ :

$$
\begin{aligned}
& \left\|(-\Delta)^{s} u^{\text {non }}(t, \cdot)\right\|_{L^{2}} \\
& \lesssim \int_{0}^{t / 2}(1+t-s)^{-\frac{n}{2(1-\rho)}\left(\frac{1}{\eta_{1}}-\frac{1}{2}\right)-\frac{s-\rho}{1-\rho}}\left\||u(s, \cdot)|^{p}\right\|_{L^{\eta_{1}} \cap L^{2}} \mathrm{~d} s \\
& +\int_{t / 2}^{t}(1+t-s)^{-\frac{s-\rho}{1-\rho}}\left\||u(s, \cdot)|^{p}\right\|_{L^{2}} \mathrm{~d} s \\
& \lesssim(1+t)^{-\frac{n}{2(1-\rho)}\left(\frac{1}{\eta_{1}}-\frac{1}{2}\right)-\frac{s-\rho}{1-\rho}} \int_{0}^{t / 2}(1+s)^{-1+\frac{n}{2(1-\rho)}\left(\frac{1}{\eta_{1}}-\frac{1}{\eta}\right)} \mathrm{d} s\|u\|_{X(T)}^{p} \\
& +(1+t)^{-1-\frac{n}{2(1-\rho)}\left(\frac{1}{\eta}-\frac{1}{2}\right)} \int_{t / 2}^{t}(1+t-s)^{-\frac{s-\rho}{1-\rho}} \mathrm{d} s\|u\|_{X(T)}^{p} \\
& \lesssim(1+t)^{-\frac{n}{2(1-\rho)}\left(\frac{1}{\eta}-\frac{1}{2}\right)-\frac{s-\rho}{1-\rho}}\|u\|_{X(T)}^{p} .
\end{aligned}
$$

We now distinguish two cases to estimate $u_{t}^{\text {non }}$. If $2<\bar{q}$, that is, $n<4 \rho \eta /(2-\eta)$ (this latter is true for any $n \geqslant 1$ if $\eta=2$ ), and

$$
\gamma_{\eta, 2}=\frac{n}{2 \rho}\left(\frac{1}{\eta}-\frac{1}{2}\right)-1,
$$

we may apply (57) with $r=\eta_{1}$ to estimate

$$
\begin{aligned}
\left\|u_{t}^{\text {non }}(t, \cdot)\right\|_{L^{2}} & \lesssim \int_{0}^{t}(1+t-s)^{-\frac{n}{2 \rho}\left(\frac{1}{\eta_{1}}-\frac{1}{2}\right)}\left\||u(s, \cdot)|^{p}\right\|_{L^{\eta_{1}} \cap L^{2}} \mathrm{~d} s \\
& \lesssim \int_{0}^{t}(1+t-s)^{-\frac{n}{2 \rho}\left(\frac{1}{\eta_{1}}-\frac{1}{2}\right)}(1+s)^{-1+\frac{n}{2(1-\rho)}\left(\frac{1}{\eta_{1}}-\frac{1}{\eta}\right)} \mathrm{d} s\|u\|_{X(T)}^{p} \\
& \lesssim(1+t)^{-\frac{n}{2 \rho}\left(\frac{1}{\eta}-\frac{1}{2}\right)}\|u\|_{X(T)}^{p},
\end{aligned}
$$

using (69) with $q=\eta_{1} p$ and with $q=2 p$. 
Now let $2 \geqslant \bar{q}$, that is, $n \geqslant 4 \rho \eta /(2-\eta)$ and

$$
\gamma_{2}=\frac{n}{2(1-\rho)}\left(\frac{1}{\eta}-\frac{1}{2}\right)-\frac{\rho}{1-\rho} .
$$

We fix $r^{*} \in(\eta, 2)$ such that

$$
\frac{n}{2}\left(\frac{1}{r^{*}}-\frac{1}{2}\right)=\rho
$$

and we apply (57) with $r=\eta_{1}$ in $[0, t / 2]$ and with $r=r^{*}$ in $[t / 2, t]$. Then, we obtain the desired estimate

$$
\begin{aligned}
& \left\|u_{t}^{\text {non }}(t, \cdot)\right\|_{L^{2}} \\
& \lesssim \int_{0}^{t / 2}(1+t-s)^{-\frac{n}{2(1-\rho)}\left(\frac{1}{\eta_{1}}-\frac{1}{2}\right)+\frac{\rho}{1-\rho}-1}\left\||u(s, \cdot)|^{p}\right\|_{L^{\eta_{1}} \cap L^{2}} \mathrm{~d} s \\
& +\int_{t / 2}^{t}(1+t-s)^{-1}\left\||u(s, \cdot)|^{p}\right\|_{L^{r^{*}} \cap L^{2}} \mathrm{~d} s \\
& \lesssim(1+t)^{-\frac{n}{2(1-\rho)}\left(\frac{1}{\eta_{1}}-\frac{1}{2}\right)+\frac{\rho}{1-\rho}-1} \int_{0}^{t / 2}(1+s)^{-1+\frac{n}{2(1-\rho)}\left(\frac{1}{\eta_{1}}-\frac{1}{\eta}\right)} \mathrm{d} s\|u\|_{X(T)}^{p} \\
& +(1+t)^{-1-\frac{n}{2(1-\rho)}\left(\frac{1}{\eta}-\frac{1}{r^{*}}\right)} \log (2+t)\|u\|_{X(T)}^{p} \\
& \lesssim(1+t)^{-\frac{n}{2(1-\rho)}\left(\frac{1}{\eta}-\frac{1}{2}\right)+\frac{\rho}{1-\rho}-1} \log (2+t)\|u\|_{X(T)}^{p},
\end{aligned}
$$

where we applied (69) with $q=r^{*} p$ and we used $p=p_{0}(n / \eta, \rho)$, to obtain

$$
\begin{aligned}
\left\||u(s, \cdot)|^{p}\right\|_{L^{r^{*}} \cap L^{2}} & \lesssim(1+s)^{-\frac{n(p-1)}{2 \eta(1-\rho)}+\frac{p \rho}{1-\rho}-\frac{n}{2(1-\rho)}\left(\frac{1}{\eta}-\frac{1}{r^{*}}\right)}\|u\|_{X(T)}^{p} \\
& =(1+s)^{-1-\frac{n}{2(1-\rho)}\left(\frac{1}{\eta}-\frac{1}{r^{*}}\right)}\|u\|_{X(T)}^{p} .
\end{aligned}
$$

Summarizing, we have proved (67), and this concludes the proof of (60). In a similar way, we prove (61) and we conclude the proof.

\subsection{Proof of Theorem 2.3}

First of all, we define the evolution space

$$
X(T)=\mathcal{C}^{1}\left([0, T], L^{\eta} \cap L^{\eta p}\right)
$$

equipped with the corresponding norm

$$
\begin{aligned}
& \|u\|_{X(T)}=\sup _{t \in[0, T]}\left((1+t)^{-1}\|u(t, \cdot)\|_{L^{\eta}}+(1+t)^{\frac{n}{2 \rho \eta}\left(1-\frac{1}{p}\right)-1}\|u(t, \cdot)\|_{L^{\eta p}}\right. \\
& \left.+\left\|u_{t}(t, \cdot)\right\|_{L^{\eta}}+(1+t)^{\frac{n}{2 \rho \eta}\left(1-\frac{1}{p}\right)}\left\|u_{t}(t, \cdot)\right\|_{L^{\eta p}}\right) .
\end{aligned}
$$

Similarly to Lemmas 4.1 and 4.2, applying the interpolation of the norms of $L^{\eta}$ and $L^{\eta p}$, we may easily prove the following lemma. 
Lemma 4.3 Let $u \in X(t)$. Then, for each $q \in[\eta, \eta p]$, it holds:

$$
\left\|u_{t}(t, \cdot)\right\|_{L^{q}} \lesssim(1+t)^{-\frac{n}{2 \rho}\left(\frac{1}{\eta}-\frac{1}{q}\right)}\|u\|_{X(t)},
$$

for any $t \geqslant 0$.

We notice that

$$
\frac{n}{2}\left(\frac{1}{\eta}-\frac{1}{\eta p}\right) \leqslant \rho
$$

as a consequence of the assumption $p \leqslant n /(n-2 \rho \eta)$ if $n \geqslant 2$ and $n=1$ with $2 \rho \eta<1$.

Setting $\mathcal{A}=L^{\eta} \cap L^{\eta p}$, as a consequence of (53) with $q=\eta$, (54) with $q=\eta p$ and $r=m=\eta$ (here we use (72)), (58) with $q=r=m=\eta$, and (58) with $q=m=\eta p$ and $r=\eta$ (here we use (72)), it follows that $u^{\text {lin }} \in X(T)$ and it satisfies

$$
\left\|u^{\operatorname{lin}}\right\|_{X(T)} \lesssim\left\|u_{1}\right\|_{\mathcal{A}} .
$$

To estimate $u^{\text {non }}$ in the space $X(T)$, we will divide the proof into two parts: for $p>p_{1}(n / \eta, \rho)$ and for $p=p_{1}(n / \eta, \rho)$. In the following discussion $j=0,1$.

Let us now consider first the supercritical case $p>p_{1}(n / \eta, \rho)$.

Using (53) with $q=\eta$ and (58) with $q=r=m=\eta$, applying (71) with $q=\eta p$, we obtain immediately

$$
\begin{aligned}
\left\|\partial_{t}^{j} u^{\text {non }}(t, \cdot)\right\|_{L^{\eta}} & \lesssim \int_{0}^{t}(1+t-s)^{1-j}\left\|\left|u_{t}(s, \cdot)\right|^{p}\right\|_{L^{\eta}} \mathrm{d} s \\
& \lesssim \int_{0}^{t}(1+t-s)^{1-j}(1+s)^{-\frac{n(p-1)}{2 \rho \eta}} \mathrm{d} s\|u\|_{X(T)}^{p} \\
& \lesssim(1+t)^{1-j}\|u\|_{X(T)}^{p},
\end{aligned}
$$

where we used Proposition 4.1 and $p>p_{1}(n / \eta, \rho)$, that is, $n(p-1)>2 \rho \eta$.

To estimate $u^{\text {non }}(t, \cdot)$ in $L^{\eta p}$, we apply (54) with $q=\eta p$ and $r=m=\eta$ (here we use (72)), so that

$$
\begin{aligned}
\left\|u^{\mathrm{non}}(t, \cdot)\right\|_{L^{\eta p}} & \lesssim \int_{0}^{t}(1+t-s)^{1-\frac{n}{2 \rho \eta}\left(1-\frac{1}{p}\right)}\left\|\left|u_{t}(s, \cdot)\right|^{p}\right\|_{L^{\eta}} \mathrm{d} s \\
& \lesssim \int_{0}^{t}(1+t-s)^{1-\frac{n}{2 \rho \eta}\left(1-\frac{1}{p}\right)}(1+s)^{-\frac{n(p-1)}{2 \rho \eta}} \mathrm{d} s\|u\|_{X(T)}^{p} \\
& \lesssim(1+t)^{1-\frac{n}{2 \rho \eta}\left(1-\frac{1}{p}\right)}\|u\|_{X(T)}^{p},
\end{aligned}
$$

where we used again Proposition 4.1 and $p>p_{1}(n / \eta, \rho)$.

The main difference with the proofs of Theorems 2.1 and 2.2 appears in the estimate of $u_{t}^{\text {non }}(t, \cdot)$ in $L^{\eta p}$. Now we apply the singular estimate (58) with $q=\eta p$ and $r=m=\eta$ (here we use (72)), so that

$$
\begin{aligned}
\left\|u^{\mathrm{non}}(t, \cdot)\right\|_{L^{\eta p}} \lesssim \int_{0}^{t} & \left((1+t-s)^{-\frac{n}{2 \rho \eta}\left(1-\frac{1}{p}\right)}+\mathrm{e}^{-c(t-s)}(t-s)^{-\frac{n}{2 \theta \eta}\left(1-\frac{1}{p}\right)}\right) \\
& \times\left\|\left|u_{t}(s, \cdot)\right|^{p}\right\|_{L^{\eta}} \mathrm{d} s .
\end{aligned}
$$


We estimate

$$
\begin{aligned}
& \int_{0}^{t}(1+t-s)^{-\frac{n}{2 \rho \eta}\left(1-\frac{1}{p}\right)}\left\|\left|u_{t}(s, \cdot)\right|^{p}\right\|_{L^{\eta}} \mathrm{d} s \\
& \lesssim \int_{0}^{t}(1+t-s)^{-\frac{n}{2 \rho \eta}\left(1-\frac{1}{p}\right)}(1+s)^{-\frac{n(p-1)}{2 \rho \eta}} \mathrm{d} s\|u\|_{X(T)}^{p} \\
& \lesssim(1+t)^{-\frac{n}{2 \rho \eta}\left(1-\frac{1}{p}\right)}\|u\|_{X(T)}^{p} .
\end{aligned}
$$

On the other hand,

$$
\frac{n}{2 \theta \eta}\left(1-\frac{1}{p}\right)<\frac{n}{2 \rho \eta}\left(1-\frac{1}{p}\right) \leqslant 1,
$$

as a consequence of (72), so that we may also estimate

$$
\begin{aligned}
& \int_{0}^{t} \mathrm{e}^{-c(t-s)}(t-s)^{-\frac{n}{2 \theta \eta}\left(1-\frac{1}{p}\right)}\left\|\left|u_{t}(s, \cdot)\right|^{p}\right\|_{L^{\eta}} \mathrm{d} s \\
& \lesssim \int_{0}^{t} \mathrm{e}^{-c(t-s)}(t-s)^{-\frac{n}{2 \theta \eta}\left(1-\frac{1}{p}\right)}(1+s)^{-\frac{n(p-1)}{2 \rho \eta}} \mathrm{d} s\|u\|_{X(T)}^{p} \\
& \lesssim(1+t)^{-\frac{n(p-1)}{2 \rho \eta}}\|u\|_{X(T)}^{p} \leqslant(1+t)^{-\frac{n}{2 \rho \eta}\left(1-\frac{1}{p}\right)}\|u\|_{X(T)}^{p},
\end{aligned}
$$

where in the last inequality we used $p>p_{1}(n / \eta, \rho)$.

Now let $p=p_{1}(n / \eta, \rho)$. Because

$$
\frac{n}{2}\left(\frac{1}{\eta}-\frac{1}{\eta p_{1}(n / \eta, \rho)}\right)=\frac{n \rho}{n+2 \eta \rho}<\rho,
$$

we may fix $\eta_{1} \in(1, \eta)$ such that

$$
\frac{n}{2}\left(\frac{1}{\eta_{1}}-\frac{1}{\eta p}\right) \leqslant \rho,
$$

and $\eta_{1} p \geqslant \eta$. It is now sufficient to follow the previous steps of the proof, replacing $\eta$ by $\eta_{1}$ and using (73) in place of (72).

For example, making use of (54) and (58), with $q=m=\eta$ and $r=\eta_{1}$, applying (71) with $q=\eta_{1} p$ and recalling that $p=p_{1}(n / \eta, \rho)$, one can derive

$$
\begin{aligned}
\left\|\partial_{t}^{j} u^{\text {non }}(t, \cdot)\right\|_{L^{\eta}} & \lesssim \int_{0}^{t}(1+t-s)^{1-j-\frac{n}{2 \rho}\left(\frac{1}{\eta_{1}}-\frac{1}{\eta}\right)}\left\|\left|u_{t}(s, \cdot)\right|^{p}\right\|_{L^{\eta_{1}}} \mathrm{~d} s \\
& \lesssim \int_{0}^{t}(1+t-s)^{1-j-\frac{n}{2 \rho}\left(\frac{1}{\eta_{1}}-\frac{1}{\eta}\right)}(1+s)^{-1+\frac{n}{2 \rho}\left(\frac{1}{\eta_{1}}-\frac{1}{\eta}\right)} \mathrm{d} s\|u\|_{X(T)}^{p} \\
& \lesssim(1+t)^{1-j}\|u\|_{X(T)}^{p},
\end{aligned}
$$

where we used Proposition 4.1 with $\alpha<1$. Similarly, we estimate $\partial_{t}^{j} u^{\text {non }}(t, \cdot)$ in $L^{\eta p}$.

Thus, we have proved (60). In the same way, we can prove (61). So, the proof is complete. 


\section{Proof of Proposition 2.1}

We only sketch the proof of Proposition 2.1, which may be easily deduced using the test function introduced in [21, 22] to modify Theorems 1, 2, 3, 4 in [13], so that they apply to fractional powers of the Laplace operator.

We define $\varphi(x)=\langle x\rangle^{-n-2 \rho}$ if $\rho>0$ and $\varphi(x)=\langle x\rangle^{-n-2 \theta}$ if $\rho=0$. Thanks to Corollary 3.3 in [21],

$$
\left|(-\Delta)^{\sigma} \varphi(x)\right| \leqslant C \varphi(x)
$$

for any $\sigma \in[\rho, 1]$ if $\rho>0$ and for any $\sigma \in[\theta, 1]$ if $\rho=0$. We also fix $\psi(t)=\chi(t)^{2 p^{\prime}}$, where $\chi \in \mathcal{C}^{\infty}([0, \infty))$, supported in $[0,1]$, nonincreasing, with $\chi=1$ in $[0,1 / 2]$. We fix $K \gg 1$ a sufficiently large constant and $R \gg 1$ a parameter, and we put $\varphi_{R}(x)=\left\langle R^{-1} K^{-1} x\right\rangle^{-n-2 \rho}$ and $\psi_{R}(t)=\psi\left(R^{-\kappa} t\right)$, for some $\kappa>0$.

First, let $j=0$. Assume, by contradiction, that $u$ is a global (in time) weak solution. Then it holds

$$
\begin{aligned}
I_{R}= & \int_{0}^{\infty} \int_{\mathbb{R}^{n}}|u(t, x)|^{p} \varphi_{R}(x) \mathrm{d} x \psi_{R}(t) \mathrm{d} t \\
= & \int_{0}^{\infty} \int_{\mathbb{R}^{n}} u(t, x)\left(\partial_{t}^{2}-\partial_{t}(-\Delta)^{\rho}-\partial_{t}(-\Delta)^{\theta}-\Delta\right)\left(\varphi_{R}(x) \psi_{R}(t)\right) \mathrm{d} x \mathrm{~d} t \\
& -\int_{\mathbb{R}^{n}} u_{1}(x) \varphi_{R}(x) \mathrm{d} x .
\end{aligned}
$$

We fix $\kappa=2(1-\rho)$. Using Hölder's inequality and Young's inequality, taking $K=1$, we may estimate

$$
\begin{aligned}
\int_{0}^{\infty} & \int_{\mathbb{R}^{n}}\left|u(t, x)\left(\partial_{t}^{2}-\partial_{t}(-\Delta)^{\rho}-\partial_{t}(-\Delta)^{\theta}-\Delta\right)\left(\varphi_{R}(x) \psi_{R}(t)\right)\right| \mathrm{d} x \mathrm{~d} t \\
& \leqslant C I_{R}^{\frac{1}{p}} R^{\frac{n+2(1-\rho)}{p^{\prime}}-2} \leqslant \frac{I_{R}}{p}+\frac{C}{p^{\prime}} R^{n+2(1-\rho)-2 p^{\prime}} .
\end{aligned}
$$

If $\eta>1$, the sign assumption (28) implies that

$$
\int_{\mathbb{R}^{n}} u_{1}(x) \varphi_{R}(x) \mathrm{d} x \geqslant 2^{-n-2 \rho} \int_{|x| \leqslant R} u_{1}(x) \mathrm{d} x \geqslant c \in R^{n\left(1-\frac{1}{\eta}\right)}(\log R)^{-1},
$$

for $R \gg 1$, so that the contradiction follows from the inequality

$$
0 \leqslant I_{R} \leqslant-c p^{\prime} \epsilon R^{n\left(1-\frac{1}{\eta}\right)}(\log R)^{-1}+C R^{n+2(1-\rho)-2 p^{\prime}},
$$

for large $R$ and $p<p_{0}(n / \eta, \rho)$. If $\eta=1$ and $p<p_{0}(n, \rho)$, then $R^{n+2(1-\rho)-2 p^{\prime}} \rightarrow 0$ as $R \rightarrow \infty$ and the inequality

$$
0 \leqslant \lim _{R \rightarrow \infty} I_{R} \leqslant-p^{\prime} \int_{\mathbb{R}^{n}} u_{1}(x) \mathrm{d} x
$$

implies the contradiction, thanks to the sign assumption (27). If $\eta=1$ and $p=p_{0}(n, \rho)$, we first deduce that $I_{R}$ is uniformly bounded, that is, $u \in L^{p}\left([0, \infty) \times \mathbb{R}^{n}\right)$. As a consequence, 


$$
\lim _{R \rightarrow \infty} \int_{0}^{\infty} \int_{\mathbb{R}^{n}} u(t, x)\left(\partial_{t}^{2}-\partial_{t}(-\Delta)^{\rho}-\partial_{t}(-\Delta)^{\theta}\right)\left(\varphi_{R}(x) \psi_{R}(t)\right) \mathrm{d} x \mathrm{~d} t=0,
$$

due to the fact that $\psi_{R}(t)=1$ in $[0, R / 2]$. It follows that

$$
0 \leqslant \lim _{R \rightarrow \infty} I_{R} \leqslant-p^{\prime} \int_{\mathbb{R}^{n}} u_{1}(x) \mathrm{d} x+C K^{n-2 p^{\prime}},
$$

and the contradiction follows for sufficiently large $K \gg 1$, thanks to (27), due to the fact that $n-2 p^{\prime}=-2(1-\rho)<0$ for $p=p_{0}(n, \rho)$.

Now let $j=1$. In this case, we fix $\kappa=2 \rho$. Assume, by contradiction, that $u$ is a global (in time) weak solution. Then it holds

$$
\begin{aligned}
I_{R}= & \int_{0}^{\infty} \int_{\mathbb{R}^{n}}\left|u_{t}(t, x)\right|^{p} \varphi_{R}(x) \mathrm{d} x \psi_{R}(t) \mathrm{d} t \\
= & \int_{0}^{\infty} \int_{\mathbb{R}^{n}} u_{t}(t, x)\left(-\partial_{t}+(-\Delta)^{\rho}+(-\Delta)^{\theta}\right)\left(\varphi_{R}(x) \psi_{R}(t)\right) \mathrm{d} x \mathrm{~d} t \\
& -\int_{0}^{\infty} \int_{\mathbb{R}^{n}} u_{t}(t, x) \Delta \varphi_{R}(x) \mathrm{d} x \Psi_{R}(t) \mathrm{d} t-\int_{\mathbb{R}^{n}} u_{1}(x) \varphi_{R}(x) \mathrm{d} x,
\end{aligned}
$$

where $\Psi$ is the compactly supported primitive of $-\psi$, namely

$$
\Psi(t)=\int_{t}^{\infty} \psi(\tau) \mathrm{d} \tau
$$

Proceeding as we did for $j=0$, we first take $K=1$ and we estimate

$$
\begin{aligned}
\int_{0}^{\infty} & \int_{\mathbb{R}^{n}}\left|u_{t}(t, x)\left(-\partial_{t}+(-\Delta)^{\rho}+(-\Delta)^{\theta}\right)\left(\varphi_{R}(x) \psi_{R}(t)\right)\right| \mathrm{d} x \mathrm{~d} t \\
& +\int_{0}^{\infty} \int_{\mathbb{R}^{n}}\left|u_{t}(t, x) \Delta \varphi_{R}(x)\right| \mathrm{d} x \Psi_{R}(t) \mathrm{d} t \\
\leqslant & C I_{R}^{\frac{1}{p}} R^{\frac{n+2 \rho}{p^{\prime}}-2 \rho} \leqslant \frac{I_{R}}{p}+\frac{C}{p^{\prime}} R^{n+2 \rho-2 \rho p^{\prime}} .
\end{aligned}
$$

If $\eta>1$, using (76), the contradiction follows from the inequality

$$
0 \leqslant I_{R} \leqslant-c p^{\prime} \epsilon R^{n\left(1-\frac{1}{\eta}\right)}(\log R)^{-1}+C R^{n+2 \rho-2 \rho p^{\prime}},
$$

for large $R$ and $p<p_{1}(n / \eta, \rho)$. If $\eta=1$ and $p<p_{1}(n, \rho)$, then $R^{n+2 \rho-2 \rho p^{\prime}} \rightarrow 0$ as $R \rightarrow \infty$ and inequality (77) implies the contradiction, thanks to the sign assumption (27). If $\eta=1$ and $p=p_{1}(n, \rho)$, we first deduce that $I_{R}$ is uniformly bounded, that is, $u_{t} \in L^{p}\left([0, \infty) \times \mathbb{R}^{n}\right)$. As a consequence,

$$
\lim _{R \rightarrow \infty} \int_{0}^{\infty} \int_{\mathbb{R}^{n}} u_{t}(t, x) \varphi_{R}(x) \mathrm{d} x \partial_{t} \psi_{R}(t) \mathrm{d} t=0,
$$

due to the fact that $\psi_{R}(t)=1$ in $[0, R / 2]$. It follows that

$$
0 \leqslant \lim _{R \rightarrow \infty} I_{R} \leqslant-p^{\prime} \int_{\mathbb{R}^{n}} u_{1}(x) \mathrm{d} x+C K^{n-2 \rho p^{\prime}},
$$


and the contradiction follows for sufficiently large $K \gg 1$, thanks to (27), due to the fact that $n-2 \rho p^{\prime}=-2 \rho<0$ for $p=p_{1}(n, \rho)$.

This concludes the proof.

\section{Concluding remarks}

Initial data are assumed to be small in Sobolev spaces, since we take advantage of the diffusion phenomenon and of the smoothing effect to obtain the desired estimates for the solution. These two crucial properties of equation (8) are related to the presence of the two dissipative terms. Assuming small, smooth initial data, possibly with compact support, could lead to gain more spatial regularity for the solution, at least for smooth nonlinearities, i.e., for large power nonlinearities. Still, we cannot expect a classical argument of well-posedness in $\mathcal{C}^{\infty}$, due to the lack of finite speed of propagation, since the fractional Laplace operator is nonlocal. We did not investigate the possible gain of regularity of solution assuming more regular data and sufficiently large power nonlinearities, since in this paper we focused on some minimal data regularity which guarantees the existence of a global-in-time solution in the energy space, for any supercritical power.

The assumption $u(0, x)=0$ in (1) may be easily replaced in Theorems 2.1,2.2 and 2.3 by the smallness of the initial data $u_{0}(x)=u(0, x)$ in an appropriate space. For instance, we may supplement (11) in the statement of Theorem 2.1 with

$$
u_{0} \in W^{2,1} \cap H^{2 \theta} \quad \text { with } \quad\left\|u_{0}\right\|_{W^{2,1} \cap H^{2 \theta}} \leqslant \epsilon .
$$

Here $W^{2,1}$ is the Sobolev space of functions in $L^{1}$ with their derivatives up to order 2. By the equivalence of the norm in Sobolev spaces and in Bessel potential spaces of positive even order, the smallness assumption in (78) implies, in particular, that

$$
u_{0},(-\Delta)^{\theta} u_{0} \in L^{1} \cap L^{2}, \quad\left\|u_{0}\right\|_{L^{1} \cap L^{2}}+\left\|(-\Delta)^{\theta} u_{0}\right\|_{L^{1} \cap L^{2}} \leqslant \epsilon .
$$

This is sufficient to replace (63) in the proof of Theorem 2.1, by

$$
\left\|u^{\operatorname{lin}}\right\|_{X(T)} \lesssim\left\|u_{0}\right\|_{W^{2,1} \cap H^{2 \theta}}+\left\|u_{1}\right\|_{L^{1} \cap L^{2}},
$$

where now

$$
u^{\text {lin }}=K_{0}(t, x) *_{(x)} u_{0}(x)+K_{1}(t, x) *_{(x)} u_{1}(x), \quad \text { with } K_{0}=\partial_{t} K_{1}+(-\Delta)^{\rho} K_{1}+(-\Delta)^{\theta} K_{1} .
$$

Similarly, in the statement of Theorem 2.2 and, respectively, Theorem 2.3 we may supplement (17) with

$$
u_{0} \in W^{2 \theta, \eta} \cap H^{2 \theta} \quad \text { with } \quad\left\|u_{0}\right\|_{W^{2 \theta, \eta} \cap H^{2 \theta}} \leqslant \epsilon
$$

and, respectively,

$$
u_{0} \in W^{2 \theta, \eta} \cap W^{2 \theta, \eta p} \quad \text { with } \quad\left\|u_{0}\right\|_{W^{2 \theta, \eta} \cap W^{2 \theta, \eta p}} \leqslant \epsilon .
$$

On the other hand, the assumption $u(0, x)=0$ plays a different role in the proof of Proposition 2.1. In particular, if $j=0$ assuming that $u(0, x)=u_{0}(x)$ the identity (75) becomes 


$$
\begin{aligned}
I_{R}= & \int_{0}^{\infty} \int_{\mathbb{R}^{n}}|u(t, x)|^{p} \varphi_{R}(x) \mathrm{d} x \psi_{R}(t) \mathrm{d} t \\
= & \int_{0}^{\infty} \int_{\mathbb{R}^{n}} u(t, x)\left(\partial_{t}^{2}-\partial_{t}(-\Delta)^{\rho}-\partial_{t}(-\Delta)^{\theta}-\Delta\right)\left(\varphi_{R}(x) \psi_{R}(t)\right) \mathrm{d} x \mathrm{~d} t \\
& -\int_{\mathbb{R}^{n}} u_{1}(x) \varphi_{R}(x) \mathrm{d} x-\int_{\mathbb{R}^{n}} u_{0}(x)\left((-\Delta)^{\rho} \varphi_{R}(x)+(-\Delta)^{\theta} \varphi_{R}(x)\right) \mathrm{d} x .
\end{aligned}
$$

If $\rho>0$ it is sufficient to assume $u_{0} \in L^{\eta}$ in order to estimate for both $\sigma=\rho$ and $\sigma=\theta$,

$$
\int_{\mathbb{R}^{n}} u_{0}(x)(-\Delta)^{\sigma} \varphi_{R}(x) \mathrm{d} x \lesssim R^{-2 \sigma} \int_{\mathbb{R}^{n}} u_{0}(x)\langle x / R\rangle^{-n-2 \rho} \mathrm{d} x \lesssim R^{n\left(1-\frac{1}{\eta}\right)-2 \sigma}\left\|u_{0}\right\|_{L^{n}},
$$

by using (74), and Hölder's inequality if $\eta>1$. In this way, the presence of the first initial datum $u_{0}$ does not invalidate the argument in the proof of Proposition 2.1, in both the cases $\eta=1$ and $\eta>1$. On the other hand, if $\rho=0$ then (27) and (28) must be, respectively, changed in the following assumptions:

$$
\begin{aligned}
& \int_{\mathbb{R}^{n}}\left(u_{0}(x)+u_{1}(x)\right) \mathrm{d} x>0 \quad \text { if } u_{0}, u_{1} \in L^{1}, \\
& u_{0}(x)+u_{1}(x) \geqslant \epsilon|x|^{-\frac{n}{n}}(\log (1+|x|))^{-1} \quad \text { for }|x| \geqslant 1 \text { if } u_{0}, u_{1} \in L^{\eta} \text { with } \eta>1 \text {. }
\end{aligned}
$$

Under such assumptions one can prove in the case $j=0$ the same results as in Proposition 2.1 with nonvanishing first initial datum. On the other hand, removing the initial datum assumption $u_{0} \equiv 0$ in the case $j=1$ raises more difficulties, whose investigation is beyond the scope of Proposition 2.1.

Acknowledgements The second and the third author are members of the Gruppo Nazionale per l'Analisi Matematica, la Probabilità e le loro Applicazioni (GNAMPA) of the Istituto Nazionale di Alta Matematica (INdAM).

Funding Open access funding provided by Università degli Studi di Bari Aldo Moro within the CRUICARE Agreement. The first author was supported by the China Postdoctoral Foundation (Grant No. 2021T140450 and No. 2021M692084).

Open Access This article is licensed under a Creative Commons Attribution 4.0 International License, which permits use, sharing, adaptation, distribution and reproduction in any medium or format, as long as you give appropriate credit to the original author(s) and the source, provide a link to the Creative Commons licence, and indicate if changes were made. The images or other third party material in this article are included in the article's Creative Commons licence, unless indicated otherwise in a credit line to the material. If material is not included in the article's Creative Commons licence and your intended use is not permitted by statutory regulation or exceeds the permitted use, you will need to obtain permission directly from the copyright holder. To view a copy of this licence, visit http://creativecommons.org/licenses/by/4.0/.

\section{References}

1. Ikehata, R., Sawada, A.: Asymptotic profile of solutions for wave equations with frictional and viscoelastic damping terms. Asympt. Anal. 98(1-2), 59-77 (2016)

2. Ikehata, R., Michihisa, H.: Moment conditions and lower bounds in expanding solutions of wave equations with double damping terms. Asympt. Anal. 114(1-2), 19-36 (2019)

3. D'Abbicco, M.: $L^{1}-L^{1}$ estimates for a doubly dissipative semilinear wave equation. NoDEA Nonlinear Differ. Equ. Appl. 24(1), 23 (2017)

4. Chen, W.: Dissipative structure and diffusion phenomena for doubly dissipative elastic waves in two space dimensions. J. Math. Anal. Appl. 486(2), 123922 (2020) 
5. D’Abbicco, M., Ikehata, R., Takeda, H.: Critical exponent for semi-linear wave equations with double damping terms in exterior domains. NoDEA Nonlinear Differ. Equ. Appl. 26(6), 56 (2019)

6. D'Abbicco, M., Ebert, M.R.: A classification of structural dissipations for evolution operators. Math. Methods Appl. Sci. 39(10), 2558-2582 (2016)

7. Yang, Han, Milani, A.: On the diffusion phenomenon of quasilinear hyperbolic waves. Bull. Sci. math. 124(5), 415-433 (2000)

8. Marcati, P., Nishihara, K.: The $L^{p}-L^{q}$ estimates of solutions to one-dimensional damped wave equations and their application to the compressible flow through porous media. J. Differ. Eq. 191, 445-469 (2003)

9. Nishihara, K.: $L^{p}-L^{q}$ estimates for solutions to the damped wave equations in 3-dimensional space and their applications. Math. Z. 244, 631-649 (2003)

10. D'Abbicco, M., Ebert, M.R.: Diffusion phenomena for the wave equation with structural damping in the $L^{p}-L^{q}$ framework. J. Differ. Equ. 256(7), 2307-2336 (2014)

11. D'Abbicco, M., Ebert, M.R.: An application of $L^{p}-L^{q}$ decay estimates to the semi-linear wave equation with parabolic-like structural damping. Nonlinear Anal. 99, 16-34 (2014)

12. D’Abbicco, M., Reissig, M.: Semilinear structural damped waves. Math. Methods Appl. Sci. 37(11), 1570-1592 (2014)

13. D’Abbicco, M., Ebert, M.R.: A new phenomenon in the critical exponent for structurally damped semilinear evolution equations. Nonlinear Anal. 149, 1-40 (2017)

14. Ikehata, R.: Asymptotic profiles for wave equations with strong damping. J. Differ. Equ. 257(6), 2159_ 2177 (2014)

15. Ikehata, R., Onodera, M.: Remarks on large time behavior of the $L^{2}$-norm of solutions to strongly damped wave equations. Differ. Integral Equ. 30(7-8), 505-520 (2019)

16. Ikehata, R., Takeda, H.: Asymptotic profiles of solutions for structural damped wave equations. J. Dynam. Differ. Equ. 31(1), 537-571 (2019)

17. Ikehata, R., Todorova, G., Yordanov, B.: Wave equations with strong damping in Hilbert spaces. J. Differ. Equ. 254(8), 3352-3368 (2013)

18. Ponce, G.: Global existence of small solutions to a class of nonlinear evolution equations. Nonlinear Anal. 9(5), 399-418 (1985)

19. Shibata, Y.: On the rate of decay of solutions to linear viscoelastic equation. Math. Methods Appl. Sci. 23(3), 203-226 (2000)

20. D'Abbicco, M.: A benefit from the $L^{\infty}$ smallness of initial data for the semilinear wave equation with structural damping. Curr. Trend. Anal. Appl. 209-2016, Trends Math., Birkhäuser/Springer, Cham (2015)

21. D'Abbicco, M., Fujiwara, K.: A test function method for evolution equations with fractional powers of the Laplace operator. Nonlinear Anal. 202, 112114 (2021). https://doi.org/10.1016/j.na.2020.112114

22. Fujiwara, K.: A note for the global nonexistence of semirelativistic equations with nongauge invariant power type nonlinearity. Math. Methods Appl. Sci. 41(13), 4955-4966 (2018)

23. Mitidieri, E., Pokhozaev, S.I.: Absence of global positive solutions of quasilinear elliptic inequalities. Dokl. Akad. Nauk 359(4), 456-460 (1998)

24. Mitidieri, E., Pohozaev, S.I.: Nonexistence of positive solutions for a systems of quasilinear elliptic equations and inequalities in $\mathbb{R}^{n}$. Dokl. Math. 59, 1351-1355 (1999)

25. Mitidieri, E., Pohozaev, S.I.: Nonexistence of weak solutions for some degenerate elliptic and parabolic problems on $\mathbb{R}^{n}$. J. Evol. Equ. 1(2), 189-220 (2001)

26. Mitidieri, E., Pohozaev, S.I.: Nonexistence of Weak Solutions for Some Degenerate and Singular Hyperbolic Problems on $\mathbb{R}^{n}$. Proc. Steklov Inst. Math. 232, 240-259 (2001)

27. Mitidieri, E., Pohozaev, S.I.: Lifespan estimates for solutions of some evolution inequalities. Differ. Equ. 45(10), 1473-1484 (2019)

28. Zhang, Q.S.: A blow-up result for a nonlinear wave equation with damping: the critical case. C. R. Acad. Sci. Paris Sér. I Math. 333(2), 109-114 (2001)

29. Segal, I.E.: Quantization and Dispersion for Nonlinear Relativistic Equations, Mathematical Theory of Elementary Particles, pp. 79-108. M. I. T. Press, Cambridge (1966)

30. Ikeda, M., Inui, T., Okamoto, M., Wakasugi, Y.: $L^{p}-L^{q}$ estimates for the damped wave equation and the critical exponent for the nonlinear problem with slowly decaying data. Commun. Pure Appl. Anal. 18, 1967-2008 (2019)

31. D’Abbicco, M., Ebert, M.R., Picon, T.H.: The critical exponent(s) for the semilinear fractional diffusive equation. J. Fourier Anal. Appl. 25, 696-731 (2019)

Publisher's Note Springer Nature remains neutral with regard to jurisdictional claims in published maps and institutional affiliations. 